\title{
Experimental investigation of an active slat for airfoil load alleviation
}

P Singh, ${ }^{1, \text { a) }}$ L Neuhaus, ${ }^{1}$ O Huxdorf, ${ }^{2} \mathrm{~J} \mathrm{Riemenschneider,}^{2} \mathrm{~J} \mathrm{Wild}^{3}{ }^{3} \mathrm{~J} \mathrm{Peinke}^{1}{ }^{\text {and }} \mathrm{M} \mathrm{Hölling}^{1}$

1) ForWind - Institute of Physics, University of Oldenburg, 26129 Oldenburg,

Germany

${ }^{2)}$ DLR - Institute of Composite Structures and Adaptive Systems, 38108 Braunschweig,

Germany

3) DLR - Institute of Aerodynamics and Flow Technology, 38108 Braunschweig,

Germany

(Dated: July 1, 2021)

1 This article discusses the utilisation of an active slat concept to reduce turbulence induced fluctuating loads 2 on an airfoil. The performance of the active slat is tested in the wind tunnel under different complex inflows $3 \quad$ created by an active grid resulting into variations in the angle of attack. Different open loop control strategies $4 \quad$ are developed to mitigate the load fluctuations on the airfoil. The aerodynamics around the airfoil is changed 5 by actively moving the trailing edge of the slat. It is observed that the active slat concept is able to alleviate KEYWORDS load fluctuations on the airfoil for inflow angle fluctuations of different scales.

7

leading edge slat, active slat, active load control, turbulence, blade unsteady load, active grid

\section{INTRODUCTION}

8 Wind turbines experience various kinds of loads in 9 their working lifetime. Their operation in the atmo10 spheric boundary layer exposes them to turbulent wind 11 fields. Turbulent structures of various scales and wind 12 gusts causing inflow velocity fluctuations interact with ${ }_{13}$ the wind turbine blades ${ }^{1,2}$. As the inflow velocity is one ${ }_{14}$ of the main components for determining the angle of at15 tack perceived by the sectional airfoils on a wind turbine ${ }_{16}$ blade, its fluctuation results in the unsteadiness in angle 17 of attack as well. This induces unsteady loads on the ${ }_{18}$ wind turbine blade which can cause fatigue damage ${ }^{3-5}$. ${ }_{19}$ In a recent study by Rezaeiha et al. ${ }^{6}$ it was found that 20 more than $65 \%$ of flapwise fatigue loads are due to tur${ }_{21}$ bulence. This is undesirable because of its deteriorating 22 effect on the blade life and efficiency, ultimately leading ${ }_{23}$ to blade structural failure ${ }^{7}$. This is why reduction of ${ }_{24}$ these loads is important for the development of efficient ${ }_{25}$ modern wind turbines and for the reduction of the cost 26 of wind energy.

${ }_{27}$ Commonly, wind turbines rely on pitch control meth28 ods such as cyclic pitch control and individual pitch con${ }_{29}$ trol (IPC) for attenuating certain loads ${ }^{8,9}$. The massive 30 inertia of the entire blade inhibits the reaction of common ${ }_{31}$ blade pitch control to the high frequency turbulence in${ }_{32}$ duced load fluctuations. Also, as the turbulent wind field 33 does not interact with all regions of the blade in the same ${ }_{34}$ way, devices which can influence local aerodynamics are 35 the requirement of modern wind turbine rotors.

${ }_{36}$ Recent years have seen the development of smart ro${ }_{37}$ tor concept through many passive and active flow con38 trol techniques which concentrate on implementation of 39 sectional devices influencing the aerodynamics in specific

\footnotetext{
${ }^{\text {a) }}$ piyush.singh@uni-oldenburg.de
}

40 regions of the blade ${ }^{10-12}$. Some of these include vortex 41 generators, trailing edge flaps, adaptive camber airfoils, ${ }_{42}$ microtabs, synthetic jets among others ${ }^{13}$. Each of these ${ }_{43}$ devices in some way or the other change the local aero44 dynamics around a region of the blade to influence the 45 loads. The trailing edge flap has become a widely re${ }_{46}$ searched control device in recent years ${ }^{14-16}$. Its popular${ }_{47}$ ity is based on the fact that it causes a shift of the lift 48 curve in the linear region, thus providing good control 49 opportunities. This makes the trailing edge flap suitable 50 for application in the outboard region of the blade. The 51 inboard region of a wind turbine blade uses thick air${ }_{52}$ foils which cannot be optimally twisted because of the 53 structural limitations of the blade, thus causing them to 54 experience early separation ${ }^{17}$. The separation can result 55 into fluctuating loads on not just the concerned thick 56 airfoil but the arising separation bubbles may also travel 57 outboard and disrupt the aerodynamic performance of 58 airfoils which have attached flow. The trailing edge flap ${ }_{59}$ is not effective in delaying the stall angle of the airfoil 60 and thus is not suitable for use in this region of the blade. ${ }_{61}$ For inboard region application, the vortex generators are ${ }_{62}$ popular passive flow control devices which help in keeping ${ }_{63}$ the flow attached to the airfoil by delaying stall ${ }^{18,19}$. The 64 last decade has also seen some work on leading edge slat 65 concepts for power performance enhancement of wind ${ }_{66}$ turbine $^{20-22}$. As compared to vortex generators leading ${ }_{67}$ edge slats have a much wider angle of attack range as well ${ }_{68}$ provide higher maximum lift values ${ }^{23,24}$. Fluctuations in 69 the inflow velocity of a wind field significantly contribute 70 to variations in the angle of attack for the airfoils in the 71 inboard region of the blade. This is due to the smaller 72 magnitude of rotational velocity caused by the proximity 73 to the axis of rotation of the wind turbine. The issue 74 with the vortex generators and fixed leading edge slats is 75 that they are fixed and cannot be controlled according to 76 the turbulence induced inflow fluctuations. In order to 77 reduce fatigue loads and to extract more energy from the 
78 root region, a flow control device is required which can 79 provide the ability to actively control the aerodynamics ${ }_{80}$ of the airfoil as well as help in avoiding flow separation. ${ }_{81}$ An actively deformable leading edge slat system for air82 foil load mitigation was recently investigated in a wind ${ }_{83}$ tunnel by Neuhaus et al. ${ }^{25}$. The work focused on the ${ }_{84}$ characterisation and estimation of the concept's initial ${ }_{85}$ capabilities. It was reported that the leading-edge ac${ }_{86}$ tive slat significantly delays the stall to higher angles of 87 attack. For a sinusoidal inflow, the active slat was able 88 to reduce $20 \%$ of the lift force fluctuations. It was also 89 reported that there is a dependency of the lift coefficient 90 on the gap size between the slat and the main body of the ${ }_{91}$ airfoil. As it was a preliminary investigation, this prop${ }_{92}$ erty was not utilised for designing the control strategy.

${ }_{93}$ The present study takes the work of Neuhaus et al. ${ }^{25}$ ${ }_{94}$ further by comprehensively gauging the performance of 95 the active slat by testing it under complex inflow con96 ditions. An active grid is used to create span-wise cor97 related inflow angle fluctuations with user-defined prop${ }_{98}$ erties like different intermittency levels ${ }^{26-28}$. The active 99 slat provides the ability to change the aerodynamic forces 100 acting on the airfoil. Different open loop control strate101 gies are designed and implemented which leverage this 102 property of the active slat, to reduce the fluctuating aero103 dynamic forces under the influence of turbulent inflow 104 conditions. The loads on the airfoil in the controlled slat 105 cases are compared to the case where the slat is static.

106 The article begins with the presentation of the experi${ }_{107}$ mental setup in section II. This section in detail discusses 108 the wind tunnel, active grid, measurement sensors and 109 most importantly the airfoil with an integrated active ${ }_{110}$ slat. The characteristics of the different turbulent inflow 111 cases is presented in section III. Section IV presents the 112 method for generating the open loop control slat trajec113 tory which is used to control the motion of the active 114 slat. This is followed by the presentation and discussion 115 of results in section V. Lastly section VI concludes the 116 article.

\section{EXPERIMENTAL SETUP}
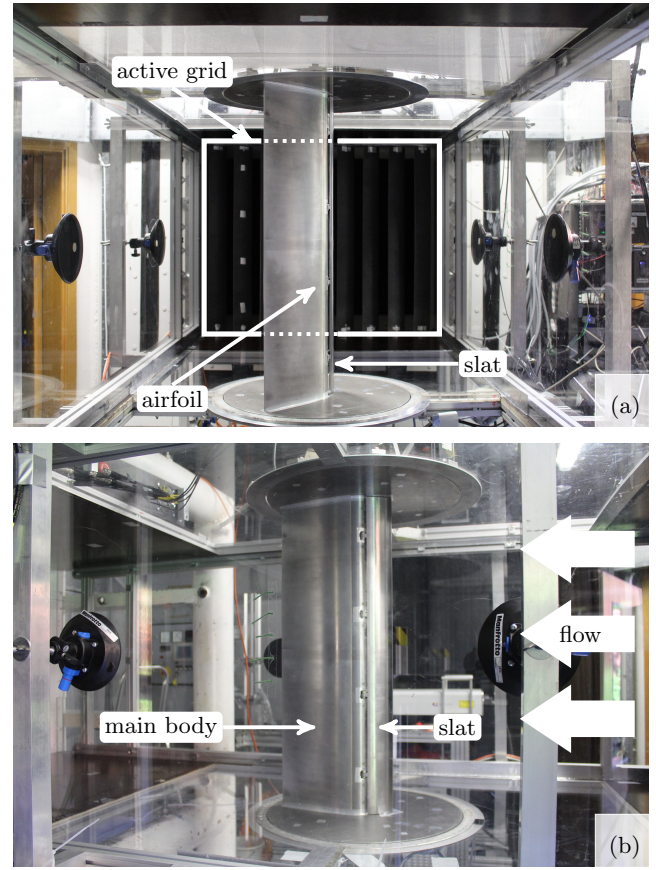

Figure 1: Active grid and airfoil with integrated active slat installed in the wind tunnel (a) and side view of the airfoil (b).

132 with the same inflow at a given point in time (figure $\left.{ }_{133} 1(\mathrm{a})\right)$. The inflow angle fluctuations generated by the ac134 tive grid are measured prior to installation of the airfoil, 135 in an empty wind tunnel. A X-type hot-wire is placed at 136 the leading edge position of the airfoil located approxi137 mately $1 \mathrm{~m}$ downstream of the active grid. The sampling 138 frequency of the hot wire measurement is $10 \mathrm{kHz}$.

139 The airfoil used in this measurement campaign has an 117 The measurements presented in this work are per- 140 integrated active slat (figure 1(b)). The airfoil's non de118 formed in the Göttingen type wind tunnel at the Uni- ${ }_{141}$ formed shape is based on the DU91-W2-250 airfoil. The 119 versity of Oldenburg. The wind tunnel has a test cross 142 design and optimisation of the integrated slat has been 120 section of $1 \mathrm{~m} \times 0.8 \mathrm{~m}$ (width and height), while it is $2.6 \mathrm{~m}{ }_{143}$ done by Manso et al. ${ }^{31}$. The airfoil has a thickness to 121 in length. Wind speeds up to $50 \mathrm{~m} \mathrm{~s}^{-1}$ can be generated 144 chord ratio of $25 \%$ with the chord being $c=300 \mathrm{~mm}$. 122 in the wind tunnel. Turbulence intensity in laminar con- ${ }_{145}$ Reynolds number of up to $\operatorname{Re}_{c}=1 \times 10^{6}$ can be achieved ${ }_{123}$ ditions have been reported to be around $0.3 \%$ by previous ${ }_{146}$ with this experimental set-up. The airfoil was tripped in ${ }_{124}$ studies in the wind tunnel ${ }^{29}$

${ }_{147}$ order trigger laminar turbulent transition and to prevent 125 The inflow angle fluctuations are generated by using 148 any separation arising from laminar separation bubble 126 a special design of an active grid ${ }^{30}$. The active grid is 149 burst. The structural design of the slat was done by 127 mounted directly at the nozzle outlet of the wind tun- ${ }_{150}$ Huxdorf et al. ${ }^{32}$. The leading edge position of the slat $128 \mathrm{nel}$ and consists of 9 vertically mounted shafts which can 151 remains fixed while the trailing edge of the slat can be 129 be controlled independently. The rectangular profile and 152 moved by deforming the slat's compliant middle section 130 parallel orientation of these shafts with respect to the ${ }_{153}$ on the pressure side using a stepper motor. For further 131 airfoil ensure that the entire span of the airfoil interacts 154 details on the active slat structural design the reader is 
155 referred to the work of Huxdorf et al. ${ }^{32}$. The movement 197 scenario, the DU25-A17 airfoil located at $45 \%$ of the ro156 of the trailing edge of the slat changes the gap size $g_{s} 198$ tor for the NREL 5 MW reference wind turbine is taken 157 between the slat and the main body of the airfoil (figure 199 into consideration ${ }^{35}$. For the realistic estimation of the 1582 ). The gap size can be varied between $g_{s} / c=1.06 \%$ to 200 angle of attack, a section of the data measured at FINO1 ${ }_{159} g_{s} / c=2.83 \%(3.18-8.49 \mathrm{~mm})$. The non deformed slat po- 201 site in North sea was taken into account. Considering an ${ }_{160}$ sition, which closely represents the clean profile is termed 202 induction factor of 0.2 and twist angle of around $8^{\circ}$ the ${ }_{161}$ as the aerodynamic reference slat position. The corre- 203 inflow data is transformed into the airfoil coordinate sys162 sponding gap size is the aerodynamic reference gap size 204 tem. The resultant angle of attack as seen by the airfoil

163 and is defined as $g_{s, r e f} / c=2.05 \%$.

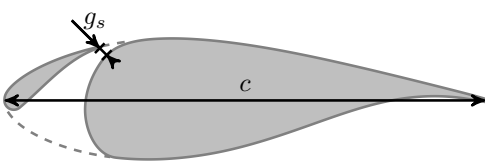

Figure 2: Investigated airfoil with controllable gap size $g_{s}$ between slat and main body of the airfoil.

164 The wind tunnel's top and bottom walls have turn ${ }_{165}$ tables which are connected to a load cell for force and 165 tables which are connected to a load cell for force and
166 torque measurements. These measurements are done at 167 a sampling frequency of $1 \mathrm{kHz}$. The airfoil is connected 2 168 to the turntable on either side of its span. The axis of ${ }_{169}$ rotation of the turntable setup is at the quarter chord 170 position of the airfoil. The airfoil is pitched about this 171 axis using a stepper motor. The pitch angle of the air172 foil is monitored using a directional sensor attached to 173 the lower turntable. The humidity and temperature of 174 the air is measured with a humidity-temperature sen175 sor while the reference wind speed is measured from the 176 dynamic pressure acquired using a differential pressure 177 transducer.

\section{COMPLEX INFLOW CHARACTERIZATION}

178 179 wind turbine directly translates into fluctuations in the 233 for the generated inflows to distinctly interact with the 180 angle of attack of the sectional airfoil. It is safe to say 234 airfoil, they should have different distribution of the re181 that any distinctive features in $u(t)$ would also migrate 235 duced frequencies. A detailed insight in the distribution ${ }_{182}$ to $\alpha(t)$. Thus in this article the turbulent inflow is char- 236 can be gained by plotting the power spectral density as 183 acterized in terms of the angle of attack variation with 237 a function of reduced frequency. The power spectra of 184 time. 238 the three inflows are plotted in figure 4. Also presented ${ }_{185}$ A wind turbine normally comes across wind fields 239 is the $1 \mathrm{P}$ reduced frequency range for a typical modern ${ }_{186}$ which are intermittent in nature. This means that the 240 wind turbine. Pereira et al. ${ }^{36}$ reported that the $1 \mathrm{P}$ re${ }_{187}$ probability of occurrence of certain extreme events are 241 duced frequency for a wind turbine can be calculated 188 higher than predicted by a Gaussian distribution ${ }^{1}$. An 242 from the local blade chord to radius ratio. For this def189 intermittent behaviour of the wind field contributes in in- 243 inition, the authors assumed that the mean velocity of ${ }_{190}$ creasing the damage equivalent load on the wind turbine 244 inflow as seen by the local airfoil is equal to the angular ${ }_{191}$ blade $^{33,34}$. A load mitigating device like the active slat 245 velocity of the airfoil. Although this is an approximation, 192 should be able to operate and perform in wind conditions ${ }_{246}$ it can provide a good estimate of the range of reduced fre${ }_{193}$ of varying levels of intermittency. Thus, to comprehen- 247 quencies associated with the interaction of natural flows 194 sively gauge the performance of the active slat, it is sub- 248 with wind turbines. Using the parameters of the DTU 10 ${ }_{195}$ jected to various wind conditions. In order to estimate ${ }_{249} \mathrm{MW}$ reference turbine ${ }^{37}$, the $1 \mathrm{P}$ reduced frequency range 196 the operating range of the angle of attack in a real world 250 for a typical modern wind turbine is calculated to be in 

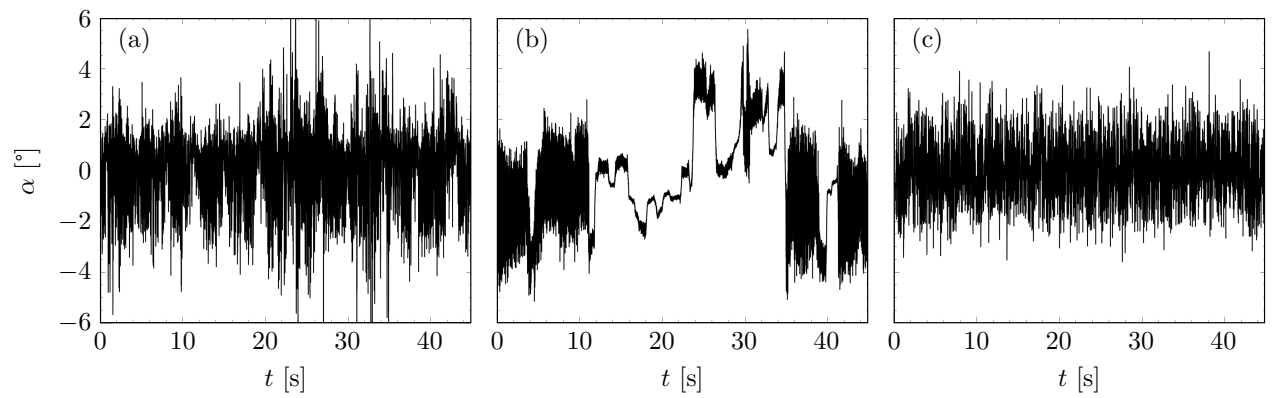

Figure 3: Turbulent inflow time series for different cases, Inflow1 (a), Inflow2 (b), Inflow3 (c)

${ }_{251}$ between $\kappa=0.004$ to $\kappa=0.17$. Leishman ${ }^{38}$ associated 252 reduced frequencies $\kappa<0.05$ to quasi steady, $\kappa>0.05$ to 253 unsteady and $\kappa>0.2$ to highly unsteady effects. Thus 254 modern wind turbines experience a broad range of un255 steady loads.

256 In figure 4 a clear difference in the energy distribution 257 over different scales are observed for the power spectra of ${ }_{285}$ is commonly used to characterise the intermittency in 259 high values for low reduced frequencies. As the reduced ${ }_{287}$ deviation of $\alpha_{\tau}$. It mainly determines the shape of the 260 frequency increases the power spectrum drops a little and ${ }_{288}$ increment PDFs. It is 0 for the Gaussian distribution and ${ }_{261}$ stabilizes in the range of $\kappa=0.05$ to $\kappa=0.5$. This ${ }_{289}$ has positive values for intermittent distribution. Higher 262 indicates that Inflow1 has significant quasi steady effects 290 values of $\lambda^{2}(\tau)$ indicate higher level of intermittency at ${ }_{263}$ along with highly unsteady components. For Inflow2 on ${ }_{291}$ the time scale $\tau$. For more details on the shape factor 264 the other hand, the energy content for the low reduced 292 and intermittency the reader is referred to the work of ${ }_{265}$ frequencies is very high and there is a high gradient in ${ }_{293}^{29}$ Castaing et al. ${ }^{39}$ and Morales et al. ${ }^{40}$ amongst others.

266 the power spectrum resulting in significantly lower energy ${ }_{294}$ The different complex inflows which are generated, ${ }_{267}$ content for larger reduced frequencies. This indicates ${ }_{295}$ aerodynamically interact with the airfoil. Thus, when 268 at the dominant presence of quasi steady effects in the 296 talking about intermittent characteristics of the flow the ${ }_{269}$ inflow. The power spectrum for Inflow3 is nearly flat 297 relevant length and time scales should be considered. As ${ }_{270}$ till $\kappa=1$. This shows that Inflow3 has white noise ${ }_{298}$ we are interested in the dynamic response of the airfoil, ${ }_{271}$ characteristics with no dominant structure present. Its 299 its chord length is used as the characteristic length. The 272 interaction with the airfoil will be largely unsteady.

300 relevant time scales for the present system can be com273 The power spectral density provides a good insight on 301 puted from the chord length and the mean wind speed ${ }_{274}$ the energy distribution over different scales but does not ${ }^{302}$ using the Taylor's hypothesis of frozen turbulence. For a 275 give any information on the time evolution of the in- 303 Reynolds number of $6 \times 10^{5}$, the time scale corresponding 276 flow. More information regarding this can be obtained 304 to the airfoil chord is about $0.01 \mathrm{~s}$. It is expected that the 277 by analysing the statistics of two temporally separated ${ }^{305}$ characteristics of the inflow with time scales higher than ${ }_{278}$ points. This helps in determining the evolution in time $3060.01 \mathrm{~s}$ would significantly influence the airfoil as well.

279 as well as provides an estimation of the intermittent 307 The PDFs of the increments inflow angle fluctuations 280 behaviour of the flow. The temporal velocity incre- ${ }^{308}$ for scale $\tau$ of $0.002 \mathrm{~s}, 0.010 \mathrm{~s}, 0.041 \mathrm{~s}, 0.167 \mathrm{~s}$ and $0.673 \mathrm{~s}$ ${ }_{281}$ ments of intermittent flows are known to display non- 309 are plotted in figure 5 (a), (c) and (e). The time scales are ${ }_{282}$ Gaussian statistics, in particular for the probability den- 310 logarithmically equidistant. The $\mathrm{X}$ axis of the increment 283 sity functions ${ }^{1}$. Deriving from the discussion above, the ${ }^{311}$ PDFs have been normalised by the standard deviation of

284 inflow angle fluctuation increments,

$$
\alpha_{\tau}(t)=\alpha(t+\tau)-\alpha(t)
$$
312 the respective inflow angle fluctuation increments. Gaus${ }_{313}$ sian PDF fits for each increment PDF have been added 314 to the plots for a comparison to the Gaussian distribu315 tion. Also presented in these figure 5 (b), (d) and (f) are 316 the shape parameter variations for the three inflows with 317 respect to the time scale $\tau$.

${ }_{318}$ When examining the behaviour of Inflow1 in figure 5 would also showcase a similar behaviour. Here $\tau$ is the ${ }_{319}$ (a), it is observed that all the PDFs corresponding to dif- 


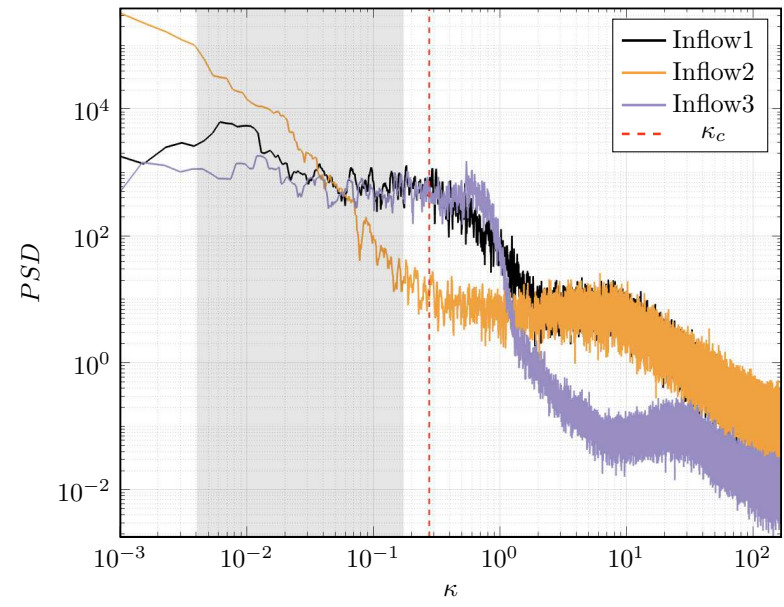

Figure 4: Power spectral density (PSD) of Inflow1, Inflow2 and Inflow3 vs reduced frequency. Shaded region represents the $1 \mathrm{P}$ reduced frequency range for a typical modern wind turbine blade. Also seen is the slat control reduced frequency $\kappa_{c}$.

\begin{abstract}
320 ferent time scales are quite similar with heavy tails and 353 tions and has gust like characteristics. The third inflow 321 exhibit non Gaussian characteristics. The corresponding 354 case Inflow3 has Gaussian characteristics for most rele322 shape factor in figure 5 (b) shows an increasing trend 355 vant scales and shows intermittent nature only for very 323 with increasing $\tau$. It reaches the maximum value at $\tau=356$ high frequency fluctuations.

$3240.015 \mathrm{~s}$, which means that the inflow displays largest in-

325 termittency levels at this time scale. Beyond $\tau=0.015 \mathrm{~s}$

326 the shape parameter reduces but still has significantly

327 high values. The increment PDFs and $\lambda^{2}$ show that

328 Inflow1 has high levels of intermittent characteristics

329 at smaller as well as larger time scales. Now focusing on 357 The definition and generation of complex inflow is fol330 inflow case Inflow2, the increment PDFs in figure 5 (c) 358 lowed by the creation of the slat trajectory through which 331 show non Gaussian distribution for all the time scales. 359 the gap size of the active slat is controlled. The static 332 For $\tau=0.673 \mathrm{~s}$ the PDF is very heavy tailed towards the 360 characterization of the active slat has been done in the 333 negative increment. The shape parameter distribution in ${ }^{361}$ previous work done at University of Oldenburg ${ }^{25}$. It was 334 figure $5(\mathrm{~d})$ shows a constant trend till approximately $\tau={ }_{362}$ found that the polar of the airfoil changes with the vari$3350.05 \mathrm{~s}$. A little drop is observed for higher time scales, 363 ation of gap size $g_{s}$ between the leading edge slat and 336 but still maintaining high $\lambda^{2}$ values. When comparing 364 the main body of the airfoil (figure 6). Thus, in principle 337 this to the shape parameter for Inflow1. Inflow2 ex- 365 providing the ability to change the aerodynamic forces 338 hibits higher values of $\lambda^{2}$ for time scales larger than $\tau=366$ acting on the airfoil for the same angle of attack $\alpha$. The $3390.02 \mathrm{~s}$. Thus, it can be inferred that this particular inflow 367 open loop control leverages this property of the active 340 has high intermittent characteristics at large time scales. 368 slat in an attempt to reduce the fluctuating aerodynamic ${ }_{341}$ The increment PDFs of inflow case Inflow3 in figure 5369 forces under the influence of complex inflow conditions. 342 (e) shows Gaussian characteristics at all the scales, ex- 370 Creation of the open loop control slat trajectory re${ }_{343}$ cept the very small time scale of $\tau=0.002 \mathrm{~s}$. This is 371 quires mainly two inputs, first being the static polar look 344 very well reflected in the shape parameter distribution in 372 up table and the second being the inflow angle time se345 figure 5 (f). The shape parameter has high values at the 373 ries. As mentioned in section III, the characterization of ${ }_{346}$ smallest time scales and sees a drastic negative gradient 374 the inflow is done by using a X-type hot wire anemome347 for higher values of $\tau$. It quickly drops to values close to 375 ter at the location of the airfoil in an empty wind tunnel. 348 0, reflecting Gaussian characteristics for large time scales. 376 The presence of the airfoil in the wind tunnel would have ${ }_{349}$ As a quick summary of the discussion above, it can 377 some effects on the flow field. In order to take these ef350 be inferred that Inflow1 consists of high as well as 378 fects into consideration for the development of the open 351 low frequency fluctuations. Inflow case Inflow2 on the 379 loop control strategies, an indirect method is used to es352 other hand displays dominating low frequency fluctua- 380 timate the angle of attack of the inflow. The forces on
\end{abstract}



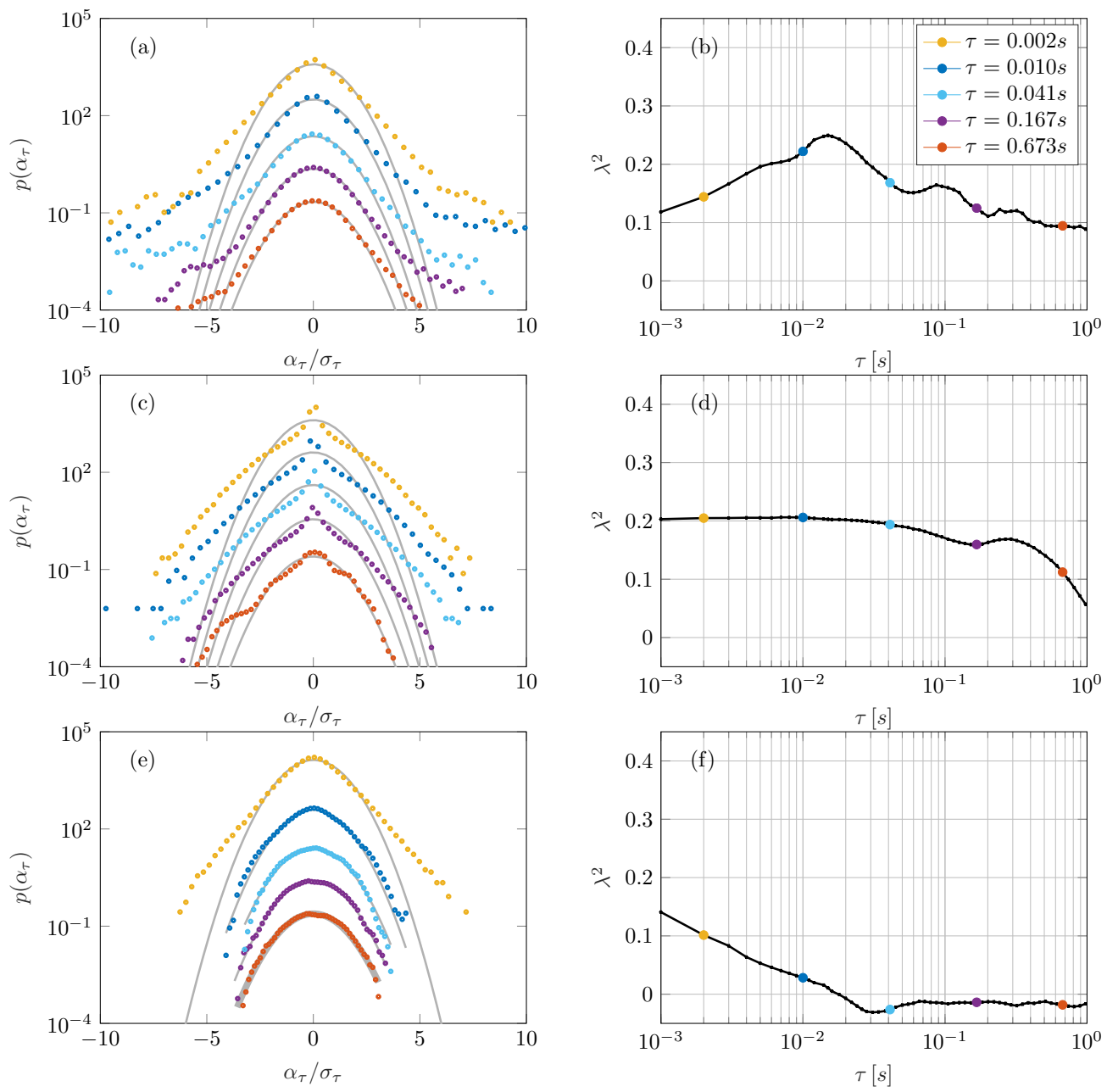

Figure 5: (a),(c), (e) show the PDF of velocity increments for Inflow1, Inflow2 and Inflow3 respectively. All graphs are vertically shifted against each other for clarity of presentation. The grey curves are respective Gaussian distribution fits. Subplots (b), (d) and (f) show the respective shape parameter $\lambda^{2}$ as a function of $\tau$ for Inflow1, Inflow2 and Inflow3.

381 the airfoil vary with time under the influence of differ- 389 tunnel. When we measure the forces we measure their 382 ent inflow. At a time instance, the measured lift coeffi- 390 average effect over the entire chord length of the airfoil. 383 cient of the airfoil is obtained. Using the lift coefficient ${ }^{391}$ Inflow structures which are very small as compared to 384 and the aerodynamic polar, the respective angle of attack 392 length scale of the airfoil get averaged out in the force 385 at that particular time instance is estimated (figure 7). ${ }_{393}$ measurements. This is why the extracted angle of attack ${ }_{386}$ When this is done for all the time instances, we get the 394 using this indirect method mostly contains the the scales 387 estimated angle of attack time series. The airfoil inter- 395 relevant to the airfoil. Leveraging the static polars for 388 acts with inflow structures of different scales in the wind 396 estimation of $\alpha(t)$ introduces a time delay due to the re- 


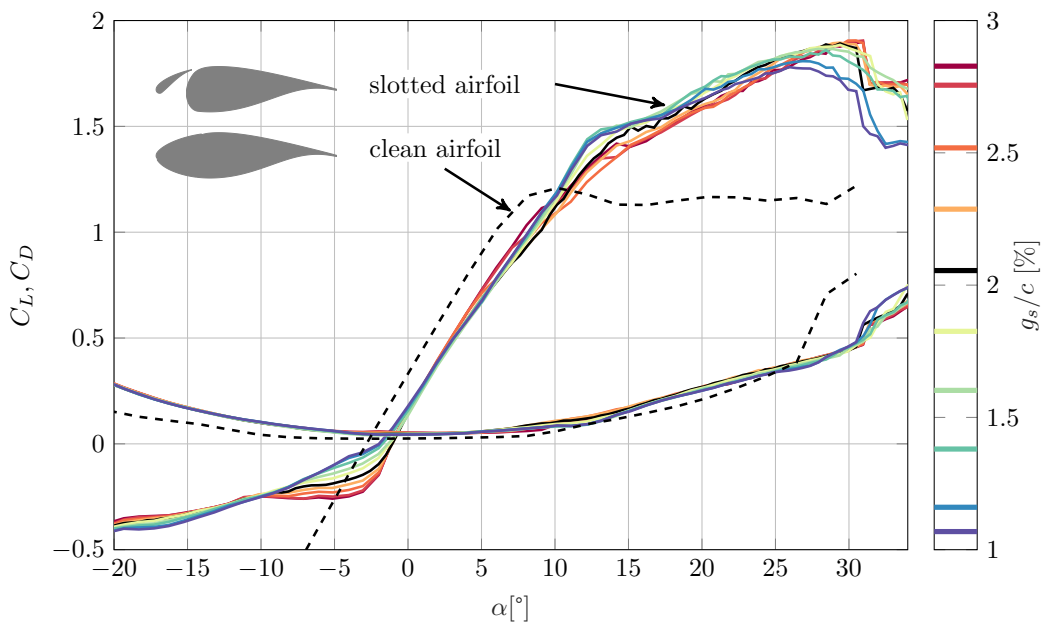

Figure 6: Static lift coefficients $C_{L}$ and drag coefficients $C_{D}$ for laminar inflow with $\operatorname{Re}=0.6 \cdot 10^{6}$ for different gap sizes $g_{s}$ compared to the clean airfoil without slat (adapted from Neuhaus et al. ${ }^{25}$ ).

397 sponse time of the airfoil to the dynamic inflow variation. 429 verse effect of inducing structural vibration in the system. 398 Having this delay in the time series itself is beneficial for ${ }_{430}$ The slat control was tested at different frequencies and 399 the slat control strategy, which can then be programmed 431 the optimum control frequency for the present scenario 400 without considering it further. 432 was found to be $8 \mathrm{~Hz}$. The results discussed in the fol${ }_{401}$ For applying this method to estimate the angle of at- ${ }_{433}^{43}$ lowing section have the slat control frequency $f_{c}$ of $8 \mathrm{~Hz}$. ${ }_{402}$ tack time series $\alpha(t)$ for a complex inflow, first the forces ${ }_{434}^{43}$ Trequency $f_{c}$ of the active slat is indicated in figure 4 . It 403 on the airfoil are measured with the slat positioned at its ${ }_{435}^{45}$ frequency $f_{c}$ of the active slat is indicated in figure 4 . It ${ }_{404}$ reference gap size $g_{s, r e f}$. The lift coefficient time series ${ }^{436}$ is observed that $\kappa_{c}$ is higher than the reduced frequen${ }_{405} C_{L}\left(t, g_{s, r e f}\right)$ and the static polar for reference gap size ${ }^{437}$ cies experienced by a typical modern wind turbine. Thus ${ }_{406} C_{L}\left(\alpha, g_{s, r e f}\right)$ acts as the input for the determination of ${ }_{439}^{438}$ wide range of unsteady characteristics.

${ }_{407} \alpha(t)$. Now based on the static polar for all $g_{s}$ and $\alpha(t)$

408 it is possible to compute $C_{L}, C_{D}$ and $C_{M}$ time series for 409 all $g_{s}$. Based on different control protocols the variation ${ }_{410}$ of slat gap-size $g_{s}(t)$ with time is obtained. The control 411 strategies can be designed in various ways to manipulate 412 the loads as desired. The details about the different con413 trol strategies used are out of scope of the present article.

\section{RESULTS} ${ }_{414}$ The gap size between the slat and mainbody of the ${ }_{442}$ stated in section III, for simulating the operating range 415 airfoil is controlled using a stepper motor as described ${ }_{443}$ of the angle of attack in a real world scenario, the airfoil 416 in section II. The gap size time series $g_{s}(t)$ needs to be ${ }_{444}$ is pitched to an angle of $10^{\circ}$. Thus the resultant angle ${ }_{417}$ translated into a control protocol which can be fed to the ${ }_{445}$ of attack $\alpha_{r}(t)$ seen by the airfoil is the summation of 418 motor. The control protocol is basically a path for the ${ }_{446}$ inflow angle time series and the airfoil pitch angle. It is 419 stepper motor to follow. This path is not a continuous 447 tested under two main cases: active slat and static slat. 420 function but rather given in discrete steps with a certain ${ }_{448}$ As the name suggests, in the active slat case the slat is 421 temporal spacing defined by a control frequency $f_{c}$. This 449 actively controlled to vary the gap size between the slat ${ }_{422}$ control frequeny can also be seen as the the frequency at 450 and main body of the airfoil. This is done according to ${ }_{423}$ which the active slat is controlled. In theory, a higher ${ }_{451}$ the designed slat control strategies. The main objective 424 control frequency should result in better load control on 452 of the designed control strategies is to reduce the fluctu425 the airfoil through manipulation of structures on a larger ${ }_{453}$ ations of the control parameters while keeping the mean 426 spectrum of scales. The control frequency however is lim- 454 value constant. The static case on the other hand refers ${ }_{427}$ ited by the torque provided by the stepper motor. The ${ }_{455}$ to the case where the gap size is fixed to $g_{s, r e f}$. This case 428 high frequency movement of the slat also causes the ad- 456 acts as the baseline case to which the active slat case is 

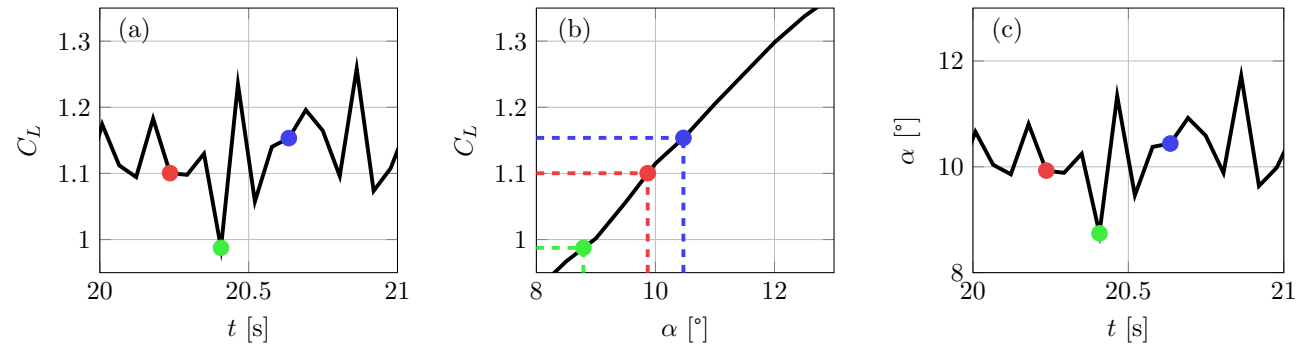

Figure 7: (a) Input $C_{L}$ time series. (b) Corresponding values of $\alpha$ from the static polar. (c) Estimated angle of attack time series.

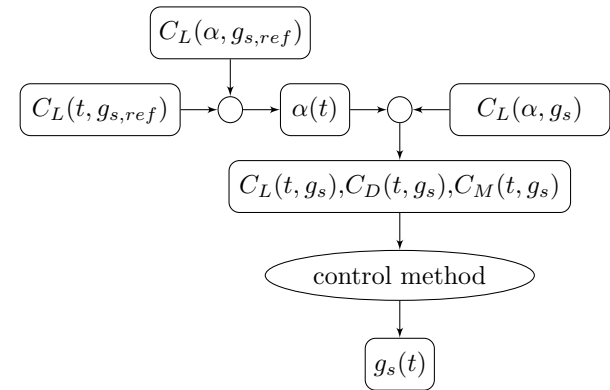

Figure 8: Slat trajectory creation algorithm

482 served that for Inflow1 the active slat is able to reduce 483 the standard deviation of the lift coefficient time series 484 by almost $10 \%$. The mean value on the other hand re485 mains almost the same for both the cases. The active 486 slat is most effective in mitigating the lift coefficient for ${ }_{487}$ the Inflow2, where a reduction in standard deviation of 488 approximately $59 \%$ is observed. For this inflow, the con489 trol strategy is able to mitigate the fluctuations caused 490 by the gust like effects in the inflow. The mean lift coeffi491 cients though shows an increase of $1.7 \%$. The active slat ${ }_{492}$ is able to mitigate most of the gust like $C_{L}$ fluctuations ${ }_{493}$ because they are created by structures with large time 494 scales.

${ }_{495}$ The active slat case seems to be ineffective in $C_{L}$ fluc496 tuation reduction for the Inflow3, rather it amplifies the ${ }_{497}$ fluctuation by almost $19 \%$. The control strategy used is 498 optimised to handle significant structures in the inflow ${ }_{457}$ compared. While the open loop control was tested for 499 as one would expect natural flows to have. The fluctua${ }_{458}$ many control parameters, the present article limits itself 500 tions due to Inflow3 are very difficult to control because 459 to the results of lift coefficient $C_{L}$ as the control param- 501 the inflow has no structures in scales which can be ac${ }_{460}$ eter. The control strategy used for the presented results ${ }_{502}$ tively manipulated by the slat. Perhaps a different con-

${ }_{461}$ aims to keep the $C_{L}$ fluctuations as low as possible with 503 trol strategy needs to be adapted to handle flows with ${ }_{462}$ respect to its mean value $\overline{C_{L}(t)}$. In this control strat- 504 white noise characteristics.

463 egy, for each time instance $t_{i}$ the gap-size providing a $C_{L}$

${ }_{464}$ value closest to $\overline{C_{L}(t)}$ is chosen as $g_{s}\left(t_{i}\right)$. This is done for

465 all the time instances to get the slat gap-size time series

${ }_{466} g_{s}(t)$.

${ }_{467}$ The effect on the lift coefficient by the airfoil's aero${ }_{468}$ dynamic interaction with the defined inflows can be ob${ }_{469}$ served in figure 9 . The figure presents a comparison of 470 the static slat and active slat cases. The control param471 eter which is used for creation of the control strategy in 472 this case is the lift coefficient. The comparison for the ${ }_{473}$ Inflow1 case in figure 9 (a) indicates a slight reduction ${ }_{506}$ necessarily mean that the total forces on the airfoil are 474 in fluctuations of the lift coefficient for the active slat. ${ }_{507}$ reduced. Positive outcome of the control can only be fully 475 When inspecting the Inflow2 case, a significant reduc- 508 judged when other coefficients are examined as well. This 476 tion in $C_{L}$ fluctuation can be observed in the active slat ${ }_{509}$ is very important because reducing fluctuations of one ${ }_{477}$ case. On the other hand the Inflow3 case does not show ${ }_{510}$ coefficient can very well result in amplification of others. 478 any observable reduction in $C_{L}$ fluctuation. 511 Also it is essential to take into account the weighted influ-

479 To get a more quantitative perspective, the values of ${ }_{512}$ ence of the reduction or amplification of each coefficient.

480 the mean and standard deviation of the $C_{L}$ time series ${ }_{513}$ For this comparative study, two approaches discussed be-

${ }_{481}$ for active and static slat is presented in table I. It is ob- ${ }_{514}$ low are being used to analyse the results further. 

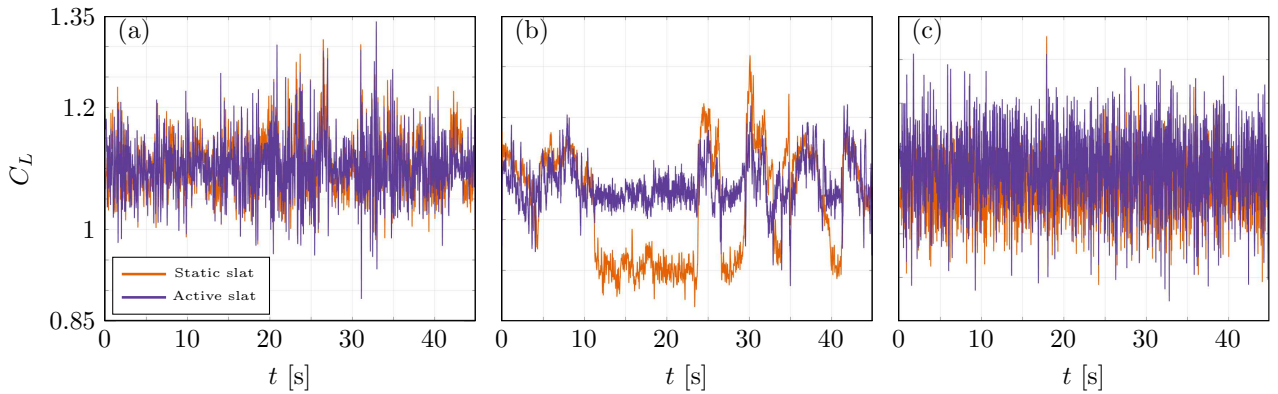

Figure 9: Lift coefficient $C_{L}$ time series comparison of the active slat (purple) and static slat with $g_{s}=g s$, ref (orange), for the inflow cases Inflow1 (a), Inflow2 (b) and Inflow3 (c).

\section{A. Cumulative standard deviation comparison}

We define a parameter,

$$
\sigma_{r}=\sqrt{\int_{0}^{f_{r}} \frac{S(f)}{f_{r}} d f}
$$

${ }_{547}$ lower as compared to the gains in the mitigation of lift ${ }_{548}$ coefficient. Inflow3 does not show any such trends and 549 the difference in the fluctuation of active and static slat 550 cases is negligible for all the coefficients.

515 which represents cumulative standard deviation. It is 551 Although the lift force fluctuation gives a good indica516 defined as the standard deviation of sum of all the com- 552 tion of the fatigue loading on the blade, a direct look at 516 ponents of a time series within the frequency range 0 to ${ }_{553}$ the resolved forces of lift and drag in the rotational plane ${ }_{518} f_{r}$ (equation (4)). Here $S$ is the power spectral density of 554 and the normal plane can give further insight. This will ${ }_{519}$ the time series. ${ }_{519}$ the time series. $\sigma_{r}$ gives an indication of the contribution ${ }_{555}$ give the resultant contribution of both the lift and drag ${ }_{521}$ Hence the standard deviation of the full time series is ${ }_{557}$ plane of the blade contributes to the edgewise force while ${ }_{522}$ equal to the cumulative standard deviation when $f_{r}$ is 558 the one in the normal plane is part of the flapwise force. ${ }_{523}$ equal to the sampling frequency of the measurement. ${ }_{559}$ We define the component of the aerodynamic forces ${ }^{524}$ Based on the definition of $\sigma_{r}$, the cumulative standard 560 (lift force $L$ and drag force $D$ ) in the flapwise force direc${ }_{525}$ deviation is computed for both the active and static slat ${ }^{561}$ tion as $F_{f}$ while the component in the edgewise direction 526 case for various values of $f_{r}$. These have been named as ${ }_{562}$ is defined as $F_{e}$ (figure 11). The time series for $F_{f}$ and $F_{e}$ ${ }_{527} \sigma_{r}$ and $\sigma_{r}$ for the respective cases. The differ- ${ }_{563}$ can be computed using the measured lift $L$ and drag $D$ ${ }_{527} \sigma_{r_{\text {active }}}$ and $\sigma_{r_{\text {static }}}$ for the respective cases. The differ- ${ }^{563}$ can time series and the total angle of attack time series $A(t)$. 529 and static cases $\left(\sigma_{r_{\text {active }}}-\sigma_{r_{\text {static }}}\right)$ is plotted with respect ${ }^{565}$ The total angle of attack $A(t)$ comprises of the resultant 529 and static cases $\left(\sigma_{r_{\text {active }}}-\sigma_{r_{\text {static }}}\right)$ is plotted with respect ${ }_{530}$ to $f_{r}$ in figure 10 . A negative value of $\left(\sigma_{r_{\text {active }}}-\sigma_{r_{\text {static }}}\right){ }_{566}$ angle of attack $\alpha_{r}(t)$ and geometrical angle $\theta$. The geo531 indicates at lower fluctuation in the active slat case as 567 metrical angle comprises of the twist and pitch angle of ${ }_{532}$ compared to the static one. On the other hand, a pos- ${ }^{568}$ the airfoil. For the present calculations $\theta$ has been set to ${ }_{533}$ itive $\left(\sigma_{r_{\text {active }}}-\sigma_{r_{\text {static }}}\right)$ indicates that the active slat is ${ }^{569} 10^{\circ}$

534 causing amplification of fluctuation as compared to that 535 of the static slat.

${ }_{536}$ Figure 10 presents the difference of the cumulative 537 standard deviation for the static and active slat cases 538 for Inflow1, Inflow2 and Inflow3. Although the con539 trol parameter for the present control strategy is the lift ${ }_{540}$ coefficient $C_{L}$, the drag $C_{D}$ and moment coefficient $C_{M}$ 541 are plotted for comparison as well. This enables us to ${ }_{542}$ understand the effect on the drag and moment penalty ${ }_{543}$ when controlling $C_{L}$. It is observed that for the Inflow1 544 and Inflow2 cases the $C_{L}$ fluctuation is significantly re-

${ }_{545}$ duced as compared to slight amplification of $C_{D}$ and $C_{M}$. 570 The respective flapwise and edgewise force coefficients ${ }_{546}$ Thus this indicates that the drag penalty is significantly 571 are termed as $C_{f}$ and $C_{e}$. They can be obtained by 

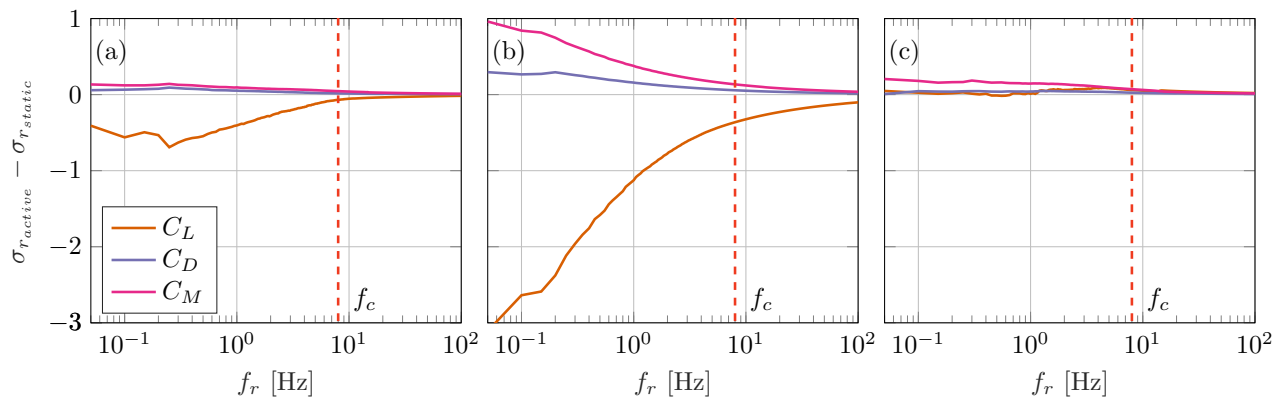

Figure 10: Difference of cumulative standard deviation of static slat $\sigma_{r_{s}}$ from active slat $\sigma_{r_{r}}$ vs $f_{r}$ for the lift coefficient $C_{L}$, drag coefficient $C_{D}$ and moment coefficient $C_{M}$. Subplots (a), (b), (c) present the inflow cases Inflow1, Inflow2 and Inflow3 respectively.

572 dividing equations 6 and 7 with $(q \cdot c \cdot s)$, where $q$ is the 584 comparison for Inflow2. Here, in both $C_{f}$ and $C_{e}$ time 573 dynamic pressure, $c$ is the airfoil chord and $s$ is the airfoil 585 series a drastic reduction of fluctuation is observed for 574 span. 586 the active slat case.

$C_{f}(t)=C_{L} \cos (A(t))+C_{D} \sin (A(t))$

$C_{e}(t)=C_{L} \sin (A(t))-C_{D} \cos (A(t))$

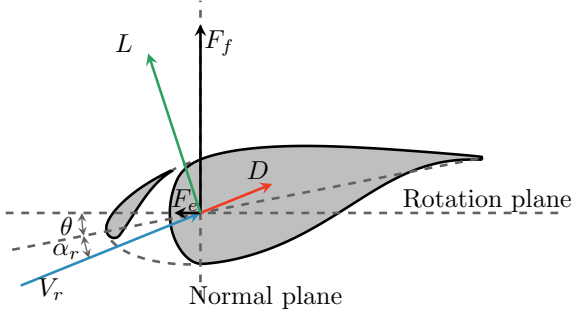

Figure 11: Forces acting on a wind turbine blade section

575 The resolved force coefficients are computed for the ${ }_{593}^{592}$ while for the edgewise component the reduction is noted 576 active and static slat cases, for each of the inflow cases 594 to be $11.5 \%$. For Inflow2 the active slat decreases the 577 defined in section III. The comparison of the active and ${ }_{595}$ fluctuating loads by $57.7 \%$ for $C_{f}$ and $15.4 \% C_{e}$. The 578 static slat case for Inflow1 is presented in figure 12, 596 active slat does not mitigate the loads for the third in579 where sub-figure 12 (a) presents the $C_{f}$ time series com- 597 flow case i.e Inflow3. Here an amplification of the loads 580 parison while sub-figure 12 (b) presents $C_{e}$ time series 598 is observed, $21.9 \%$ for $C_{f}$ and approximately $11.1 \% C_{e}$. 581 comparison. Visually a slight reduction in the fluctua- ${ }_{599}$ The load reduction with the help of the active slat in the 582 tion is observed in the active slat case for both the com- 600 edgewise direction is far less than that observed in the 583 ponents. Sub-figures 12 (c) and(d) present the respective 601 flapwise direction. It is important to remember that the 

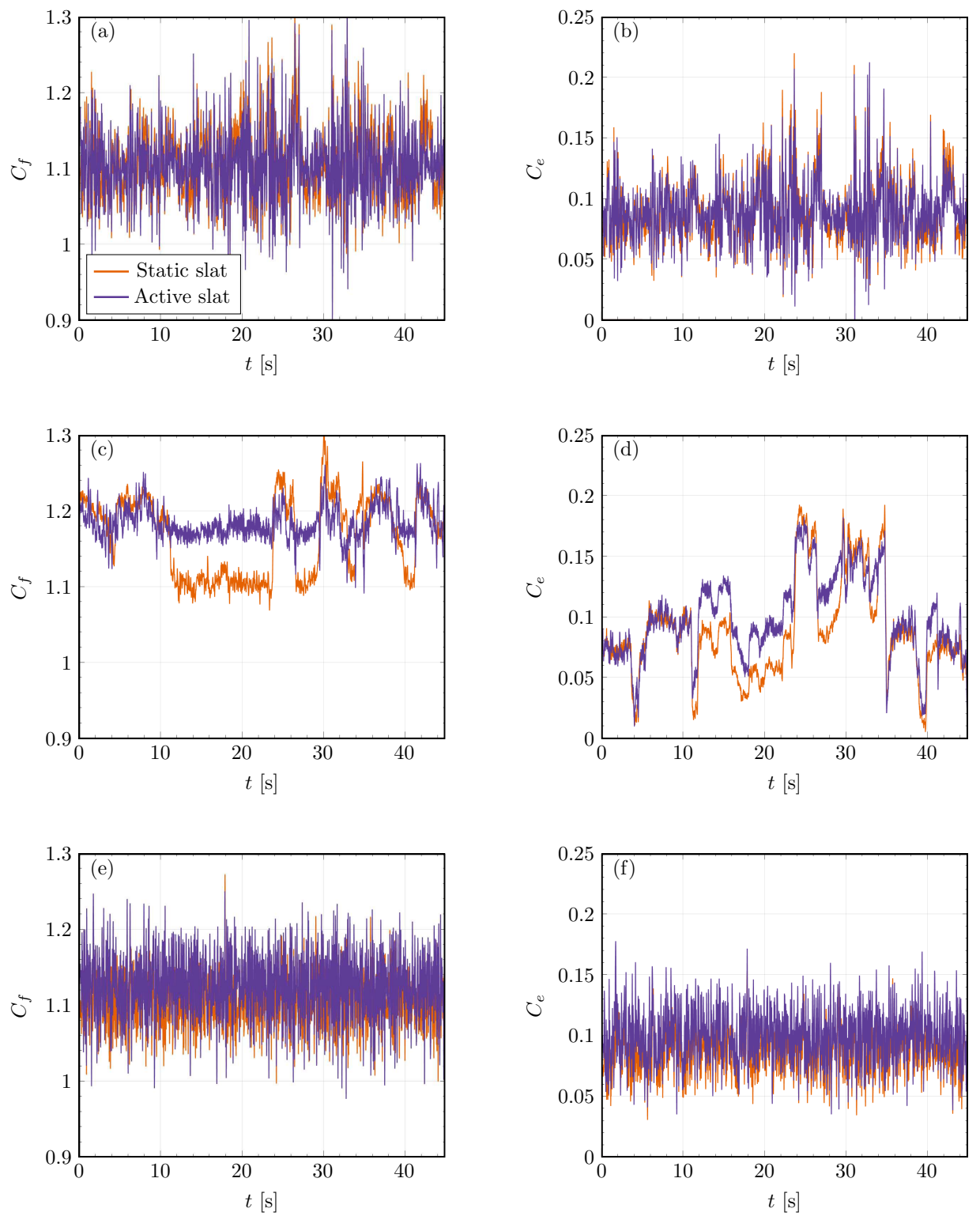

Figure 12: Subfigure(a),(c) and (e) are the $C_{f}$ time series for the inflow cases Inflow1.Inflow2 and Inflow3. Subfigure (b),(d) and (f) are the $C_{e}$ time series for the inflow cases Inflow1,Inflow2 and Inflow3. Each subfigure presents comparison of the active slat (purple) and static slat with $g_{s}=g s$, ref (orange) 
${ }_{602}$ turbulence induced fatigue loads play a very significant 656 edgewise loads respectively. For inflow case Inflow2 the ${ }_{603}$ role in the flapwise cyclic loads while the edgewise loads 657 active slat caused reductions of approximately $58 \%$ flap604 are dominated by gravitational forces ${ }^{6}$. The reduction 658 wise and $15 \%$ edgewise load fluctuations. It is ineffective 605 of turbulence induced loads in the flapwise direction is 659 in reducing the load fluctuations for Inflow3.

606 more important from the perspective of the overall load ${ }_{660}$ The experimental investigation of the active slat con607 reduction on a wind turbine blade.

\section{CONCLUSION}

608 An actively deformable integrated slat concept on a ${ }_{609}$ DU91-W2-250 airfoil was comprehensively tested for mit${ }_{610}$ igation of fluctuating loads on the airfoil. The aerody611 namic forces acting on the airfoil can be manipulated by ${ }_{612}$ changing the gap size between the slat and main body ${ }_{613}$ of the airfoil. To extensively test the active slat system, 614 three distinct complex inflow conditions of varying lev${ }_{615}$ els of intermittency were generated. Inflow case Inflow1 616 has significant presence of fluctuation at large as well as 617 small time scales. On the other hand Inflow2 has dom${ }_{618}$ inant presence of low frequency gust like features, while 619 Inflow3 has Gaussian characteristics on large scales and 620 high intermittency on small scales. The distinct features ${ }_{621}$ of the inflows ensures that the operation of the active slat 622 is investigated under a wide spectrum of loads.

${ }_{623}$ Different open loop control strategies were developed 624 to reduce the fluctuations of the desired control param${ }_{625}$ eter by keeping the variation of its mean value to the ${ }_{626}$ minimum. The present article limits itself to the discus${ }_{627}$ sion of the control parameter lift coefficient. The airfoil ${ }_{628}$ was exposed to the three inflows and the slat was actively 682 of Economic Affairs and Energy (BMWi) on decision of ${ }_{629}$ controlled for load mitigation and this case was termed ${ }_{683}$ the German Parliament in the frame of the SmartBlades ${ }_{630}$ as the active slat case. The active slat measured load 6842.0 project (funding reference no. 0324032A/D). The ${ }_{631}$ was compared to the static slat case, where the slat is 685 authors gratefully acknowledge the German Ministry of ${ }_{632}$ fixed with reference gap size. For the inflow case Inflow1 ${ }_{686}$ Economic Affairs and Energy for funding this work. The ${ }_{633}$ the active slat was able to reduce the standard deviation 687 authors are also thankful to the learned referees for their ${ }_{634}$ of the lift coefficient by $10 \%$. The active slat reduced 688 valuable constructive suggestions which has helped in im635 the fluctuating lift coefficient for Inflow2 by an astound- 689 proving this article.

${ }_{636}$ ing $59 \%$. It successfully mitigated the load fluctuations ${ }_{637}$ caused by the low frequency gust characteristics of the 638 inflow. For Inflow3 however, the active slat was ineffec${ }_{639}$ tive and rather amplified the lift coefficient fluctuation 640 by $19 \%$. This might be because the control strategy was ${ }_{641}$ optimised to handle defined structures in the inflow and ${ }_{642}$ Inflow3 is devoid of those at the scales controlled by the ${ }_{643}$ slat. For all the three inflow cases, the mean value vari${ }_{644}$ ation was kept below $2 \%$. The effect of the active slat 645 on loads other than the control parameter $C_{L}$ was inves${ }_{646}$ tigated by using the cumulative standard deviation. A ${ }_{647}$ small drag and moment penalty was observed for inflow 648 cases Inflow1 and Inflow2, but the gains obtained by ${ }_{649}$ lift fluctuation mitigation were found to be much more 650 significant. The effect of the active slat on the loads in 651 the rotational and normal planes of a wind turbine ro${ }_{652}$ tor blade was examined by resolving the lift and drag 653 forces on the airfoil in edgewise and flapwise directions. ${ }_{654}$ When exposed to Inflow1, the active slat alleviated ap655 proximately $10 \%$ and $5 \%$ fluctuations for flapwise and ${ }_{664}$ unsteady loads, but it is most effective in mitigating low 665 frequency gust like loads. It proves to be an important 666 initial step for the development of a promising active flow 67 control device for addressing the issues of energy loss due ${ }_{669}$ region of a wind turbine blade. The transition from the 670 proof of concept on a two dimensional airfoil to its ap671 plication on a three dimensional blade brings some chal${ }_{672}$ lenges which need further research. The reduction of the 673 complexity of the system is one such challenge. Other as674 pect that needs further research is the aeroacoustic noise 675 generated from the slat. The current study was based on 676 an open loop control strategy and relies on good quality 677 inflow data. Closed loop control strategies or a combina678 tion of open and closed loop control strategies need to be 679 explored to make the active slat system more robust for 680 real world operational conditions.

\section{ACKNOWLEDGMENTS}

\section{DATA AVAILABILITY}

Raw data were generated in the wind tunnel at the 691 university of Oldenburg. Derived data supporting the 692 findings of this study are available from the correspond${ }_{693}$ ing author upon reasonable request.

\section{REFERENCES}

${ }^{1}$ M. Wächter, H. Heißelmann, M. Hölling, A. Morales, P. Milan, T. Mücke, J. Peinke, N. Reinke, and P. Rinn, "The turbulent nature of the atmospheric boundary layer and its impact on the wind energy conversion process," Journal of Turbulence 13, N26 (2012).

${ }^{2} \mathrm{M}$. Hölling, J. Peinke, and S. Ivanell, Wind energy-Impact of M. Hölling, J. Peinke, a
turbulence, Vol. 2 (2014).

turbulence, Vol. 2 (2014).
${ }^{3}$ S. Lee, M. J. Churchfield, P. J. Moriarty, J. Jonkman, and

J. Michalakes, "A numerical study of atmospheric and wake tur${ }_{661}$ cept demonstrates the potential of the concept for mit${ }_{662}$ igating unsteady loads on an airfoil. The active slat is ${ }_{663}$ able to alleviate load fluctuations over a wide spectrum of 
bulence impacts on wind turbine fatigue loadings," Journal of Solar Energy Engineering 135 (2013).

${ }^{4}$ A. Lavely, G. Vijayakumar, M. Kinzel, J. Brasseur, and E. Paterson, "Space-time loadings on wind turbine blades driven by atmospheric boundary layer turbulence," in 49th AIAA Aerospace Sciences Meeting including the New Horizons Forum and Aerospace Exposition (2011)

${ }^{5}$ B. Ernst and J. R. Seume, "Investigation of site-specific wind field parameters and their effect on loads of offshore wind turbines," Energies 5, 3835-3855 (2012)

${ }^{6}$ A. Rezaeiha, R. Pereira, and M. Kotsonis, "Fluctuations of angle of attack and lift coefficient and the resultant fatigue loads for a large horizontal axis wind turbine," Renewable Energy 114 904-916 (2017).

${ }^{7}$ F. Spinato, P. J. Tavner, G. J. W. Van Bussel, and E. Koutoulakos, "Reliability of wind turbine subassemblies," IET Renewable Power Generation 3, 387-401 (2009).

${ }^{8}$ B. L. Jones, W. H. Lio, and J. A. Rossiter, "Overcoming fundamental limitations of wind turbine individual blade pitch control with inflow sensors," Wind Energy 21, 922-936 (2018).

${ }^{9}$ C. L. Bottasso, A. Croce, C. E. D. Riboldi, and M. Salvetti, "Cyclic pitch control for the reduction of ultimate loads on wind turbines," Journal of Physics: Conference Series 524, 012063 (2014).

${ }^{10}$ I. Aramendia, U. Fernandez-Gamiz, J. A. Ramos-Hernanz, J. Sancho, J. M. Lopez-Guede, and E. Zulueta, "Flow control devices for wind turbines," Energy Harvesting and Energy Efficiency , 629-655 (2017)

${ }^{11}$ T. K. Barlas and G. A. M. van Kuik, "Review of state of the art in smart rotor control research for wind turbines," Progress in Aerospace Sciences 46, 1-27 (2010).

${ }^{12}$ C. P. Van Dam, D. E. Berg, and S. J. Johnson, "Active load control techniques for wind turbines," Tech. Rep. (Sandia National Laboratories, 2008).

${ }^{13}$ S. J. Johnson, J. P. Baker, C. P. Van Dam, and D. Berg, "An overview of active load control techniques for wind turbines with an emphasis on microtabs," Wind Energy 13, 239-253 (2010).

${ }^{14}$ P. B. Andersen, L. Henriksen, M. Gaunaa, C. Bak, and T. Buhl "Deformable trailing edge flaps for modern megawatt wind turbine controllers using strain gauge sensors," Wind Energy $\mathbf{1 3}$ 193-206 (2010).

${ }^{15}$ W. Zhang, Y. Wang, R. Liu, H. Liu, and X. Zhang, "Unsteady aerodynamic modeling and control of the wind turbine with trailing edge flap," Journal of Renewable and Sustainable Energy 10 $063304(2018)$

${ }^{16} \mathrm{X}$. Bofeng, F. Junheng, L. Qing, X. Chang, Z. Zhenzhou, and Y. Yue, "Aerodynamic performance analysis of a trailing-edge flap for wind turbines," Journal of Physics: Conference Series 1037, 022020 (2018).

17I. Herráez, B. Akay, G. J. W. van Bussel, J. Peinke, and B. Stoevesandt, "Detailed analysis of the blade root flow of a horizontal axis wind turbine," Wind Energy Science 1, 89-100 (2016)

${ }^{18}$ L. Gao, H. Zhang, Y. Liu, and S. Han, "Effects of vortex generators on a blunt trailing-edge airfoil for wind turbines," Renewable Energy 76, 303-311 (2015).

${ }^{19}$ N. Troldborg, F. Zahle, and N. N. Sørensen, "Simulations of wind turbine rotor with vortex generators," Journal of Physics: Conference Series 753, 022057 (2016).

${ }^{20}$ G. Pechlivanoglou, C. N. Nayeri, and C. O. Paschereit, "Fixed leading edge auxiliary wing as a performance increasing devic for hawt blades," DEWEK, Bremen, Germany (2010).

${ }^{21}$ F. Zahle, M. Gaunaa, N. N. Sørensen, and C. Bak, "Design and wind tunnel testing of a thick, multi-element high-lift airfoil," in Proceedings of EWEA 2012 - European Wind Energy Conference and Exhibition European Wind Energy Association (EWEA) (2012)
${ }^{22}$ M. Gaunaa, F. Zahle, N. N. Sørensen, and C. Bak, "Quantification of the effects of using slats on the inner part of a $10 \mathrm{MW}$ rotor," in European Wind Energy Conference (2012).

${ }^{23}$ A. M. O. Smith, "High-lift aerodynamics," Journal of Aircraft 12, 501-530 (1975)

${ }^{24}$ D. N. Foster, "Flow around wing sections with high-lift devices," Journal of Aircraft 9, 205-210 (1972).

${ }^{25}$ L. Neuhaus, P. Singh, T. Homeyer, O. Huxdorf, J. Riemenschneider, J. Wild, J. Peinke, and M. Hölling, "Mitigating loads by means of an active slat," Journal of Physics: Conference Series 1037, 022032 (2018).

${ }^{26} \mathrm{P}$. Knebel, A. Kittel, and J. Peinke, "Atmospheric wind field conditions generated by active grids," Experiments in fluids 51, 471-481 (2011)

${ }^{27}$ L. Kröger, J. Frederik, J. W. van Wingerden, J. Peinke, and M. Hölling, "Generation of user defined turbulent inflow conditions by an active grid for validation experiments," Journal of Physics: Conference Series 1037, 052002 (2018).

${ }^{28}$ L. Kröger, L. Neuhaus, J. Peinke, G. Gülker, and M. Hölling, "Turbulence generation by active grids," in Progress in Turbulence VIII (2019)

${ }^{29}$ H. Heißelmann, J. Peinke, and M. Hölling, "Experimental airfoil characterization under tailored turbulent conditions," Journal of Physics: Conference Series 753, 072020 (2016).

${ }^{30}$ T. T. B. Wester, J. Krauss, L. Neuhaus, A. Hölling, G. Gülker, M. Hölling, and J. Peinke, "How to design a 2D active grid for dynamic inflow modulation," arXiv preprint arXiv:2012.10245 (2020)

${ }^{31}$ A. Manso Jaume and J. Wild, "Aerodynamic design and optimization of a high-lift device for a wind turbine airfoil," New Results in Numerical and Experimental Fluid Mechanics X , 859869 (2016).

${ }^{32} \mathrm{O}$. Huxdorf, J. Riemenschneider, P. Lorsch, and M. Radestock, "Structural design and experimental investigations of a shapeadaptive slat for wind energy rotor blades," in SMART2017-8th ECCOMAS Thematic Conference on Smart Structures and Materials (2017).

${ }^{33}$ C. M. Schwarz, S. Ehrich, R. Martin, and J. Peinke, "Fatigue load estimations of intermittent wind dynamics based on a blade element momentum method," Journal of Physics: Conference Series 1037, 072040 (2018).

${ }^{34}$ C. M. Schwarz, S. Ehrich, and J. Peinke, "Wind turbine load dynamics in the context of turbulence intermittency," Wind Energy Science 4, 581-594 (2019)

${ }^{35}$ J. Jonkman, S. Butterfield, W. Musial, and G. Scott, "Definition of a 5-MW reference wind turbine for offshore system development," Tech. Rep. (National Renewable Energy Lab.(NREL), Golden, CO (United States), 2009).

${ }^{36}$ G. Pereira, R.and Schepers and M. D. Pavel, "Validation of the Beddoes-Leishman dynamic stall model for horizontal axis wind turbines using MEXICO data," Wind Energy 16, 207-219 (2013)

${ }^{37}$ C. Bak, F. Zahle, R. Bitsche, T. Kim, A. Yde, L. C. Henriksen, M. H. Hansen, J. P. A. A. Blasques, M. Gaunaa, and A. Natarajan, "The DTU 10-MW reference wind turbine," in Danish Wind Power Research (2013).

${ }^{38} \mathrm{~J}$. Leishman, Principles of Helicopter Aerodynamics, Cambridge Aerospace Series (Cambridge University Press, 2016).

${ }^{39}$ B. Castaing, Y. Gagne, and E. J. Hopfinger, "Velocity probability density functions of high Reynolds number turbulence," Physica D: Nonlinear Phenomena 46, 177-200 (1990).

${ }^{40}$ A. Morales, M. Wächter, and J. Peinke, "Characterization of wind turbulence by higher-order statistics," Wind Energy 15, 391-406 (2012) 


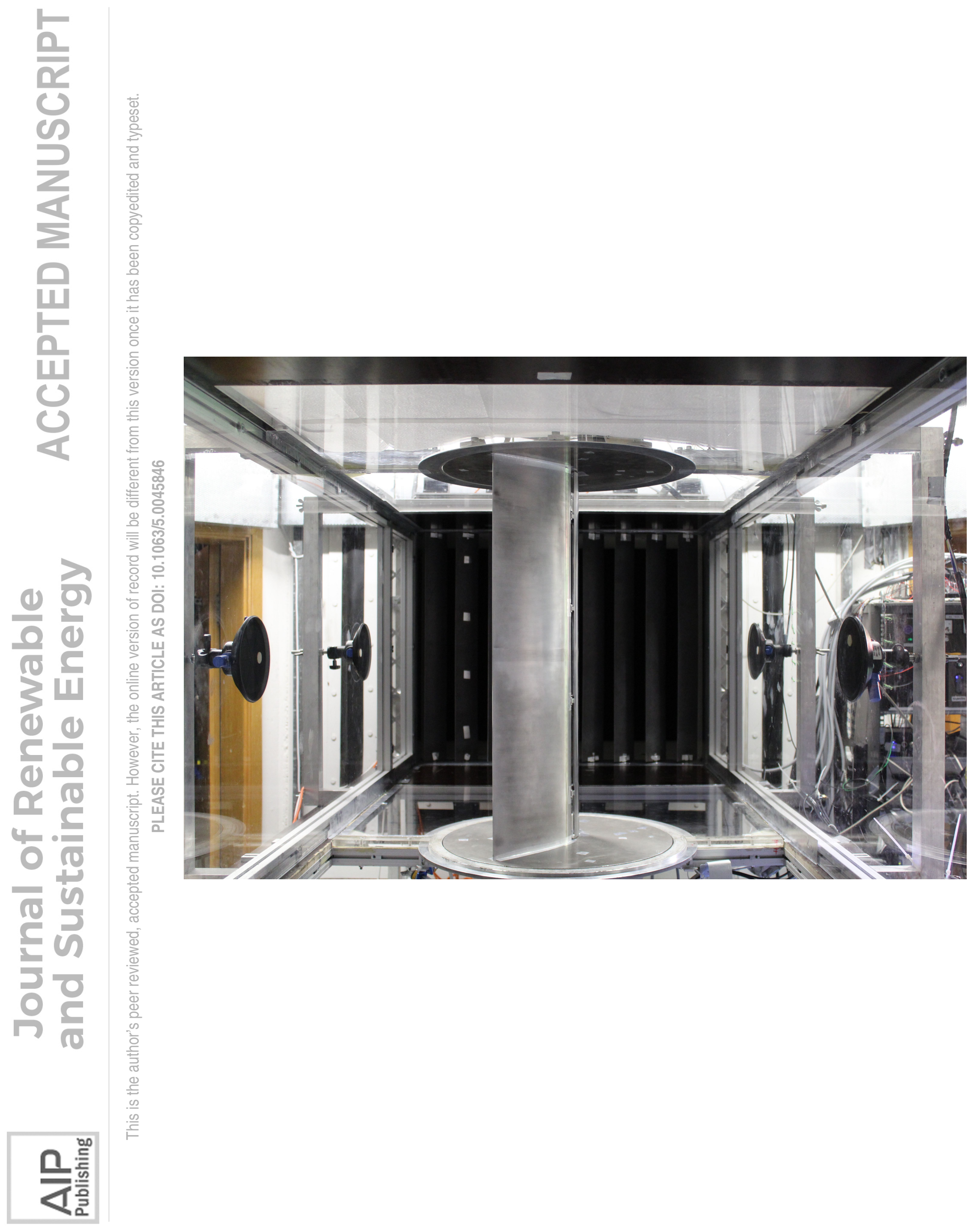




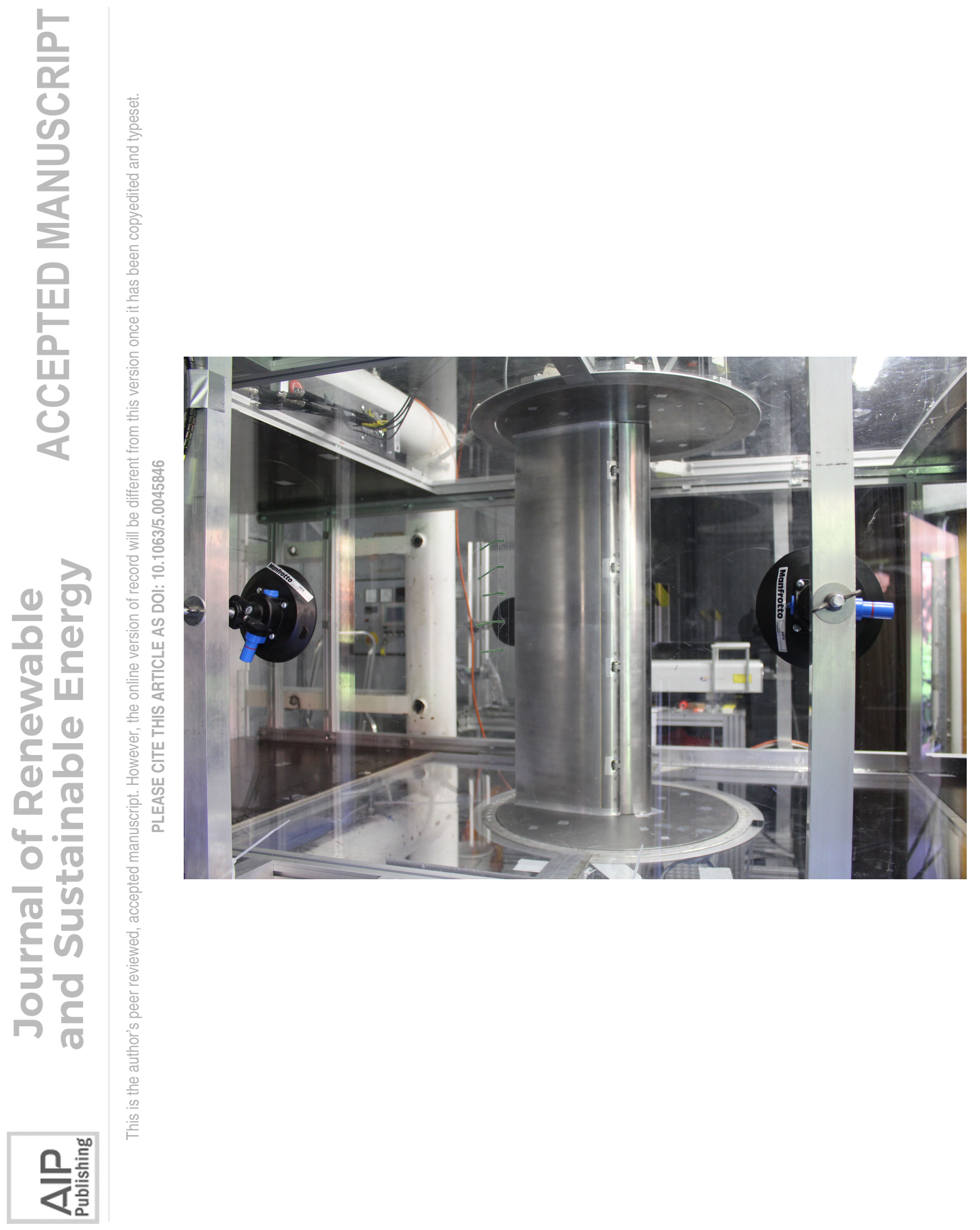



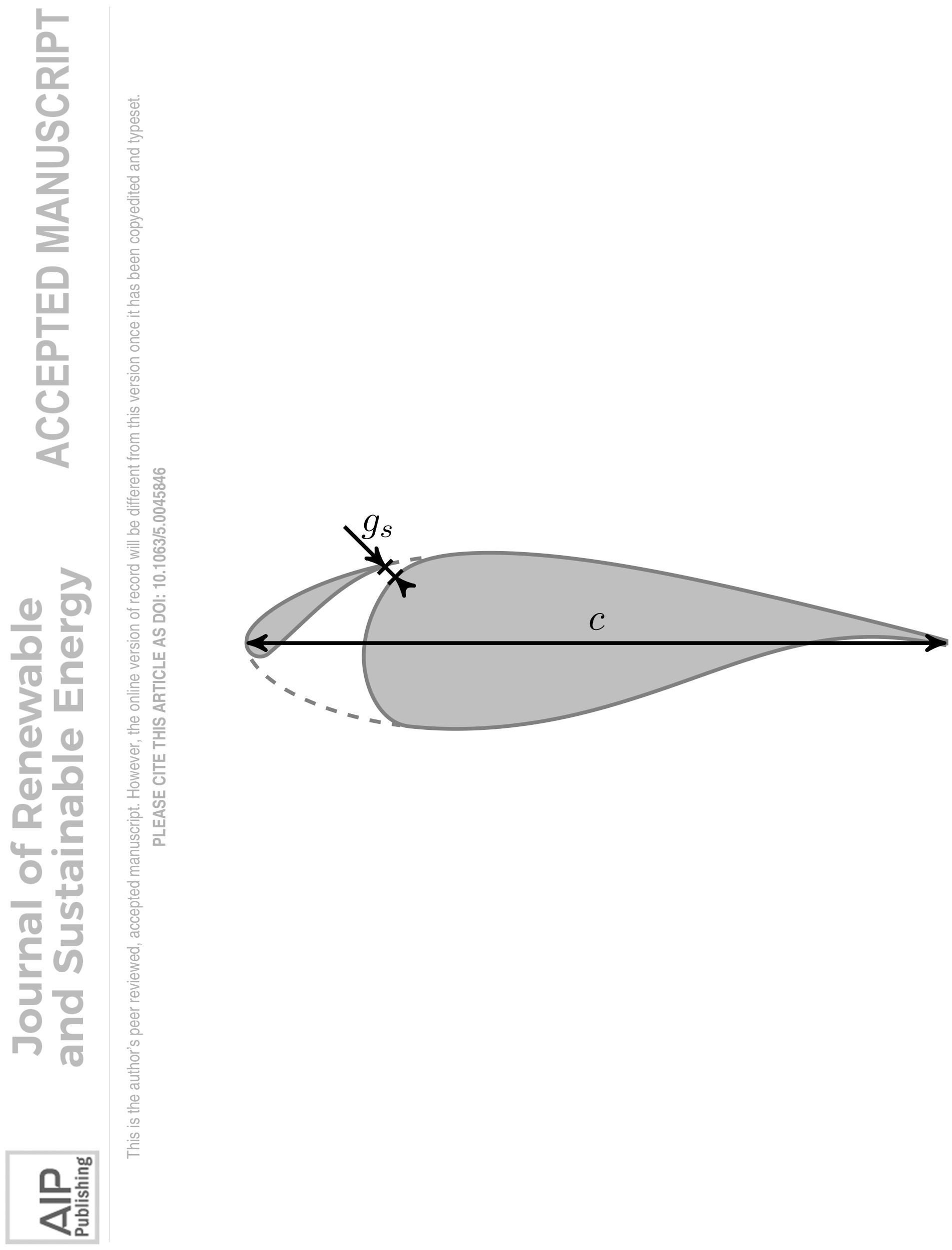


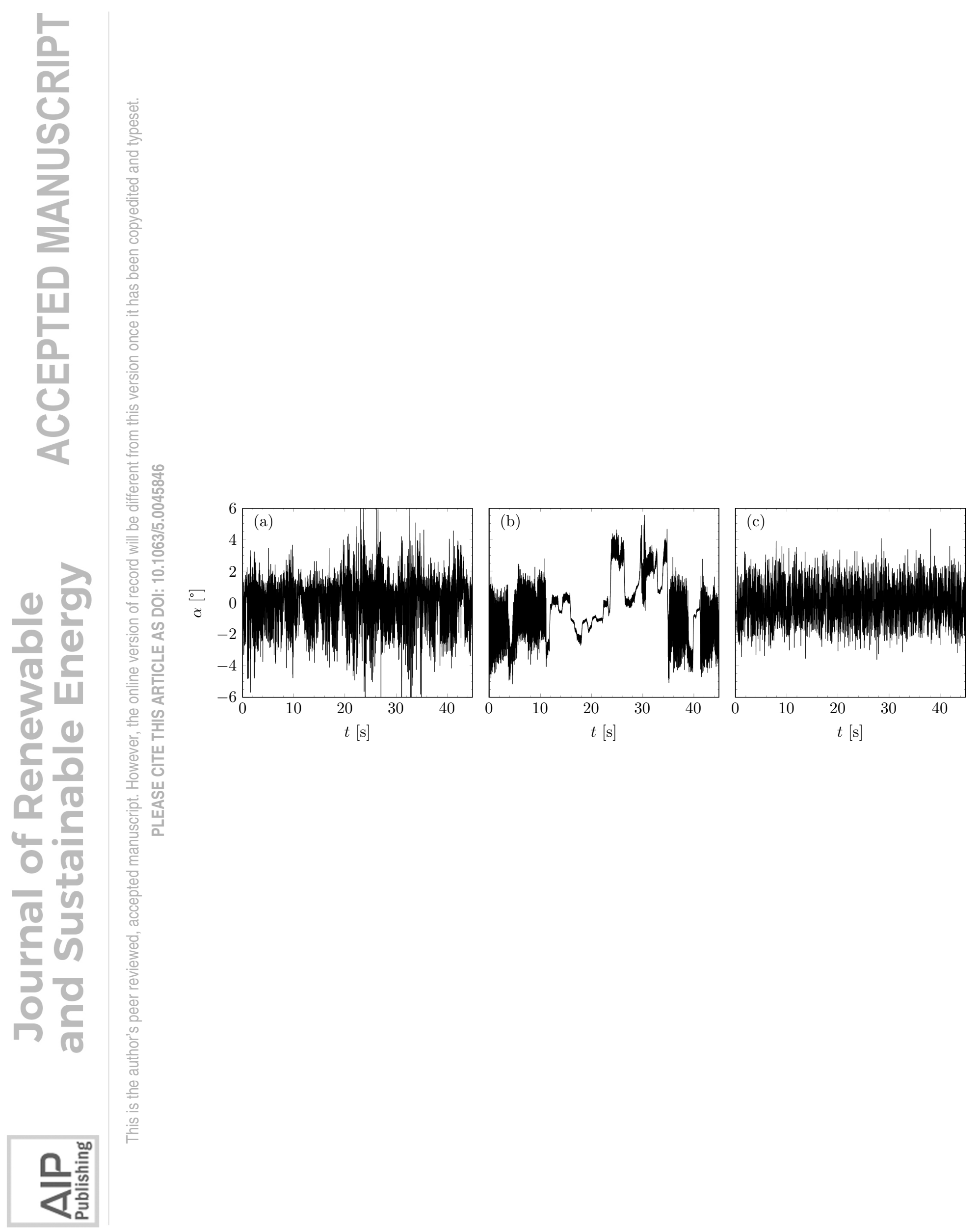




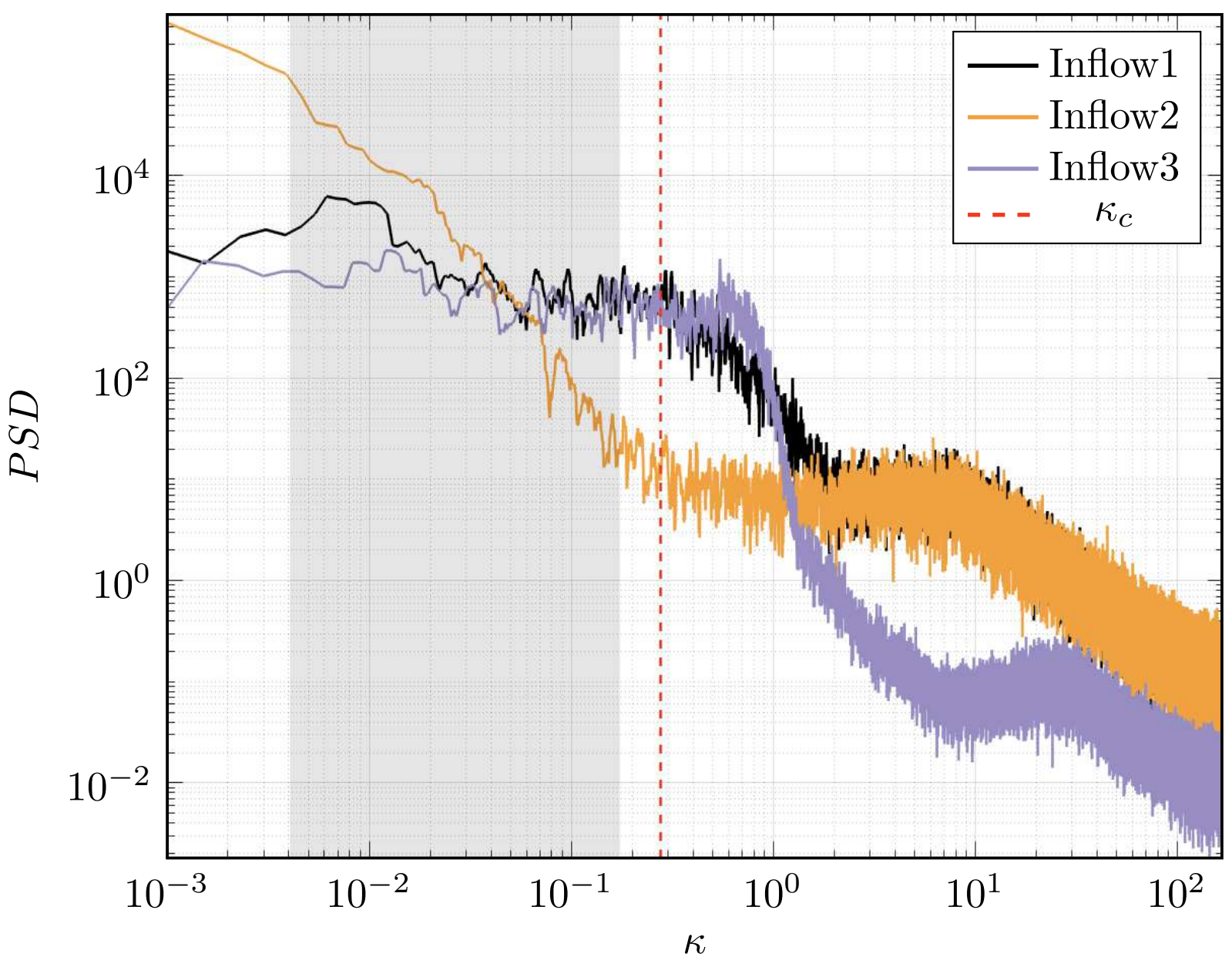



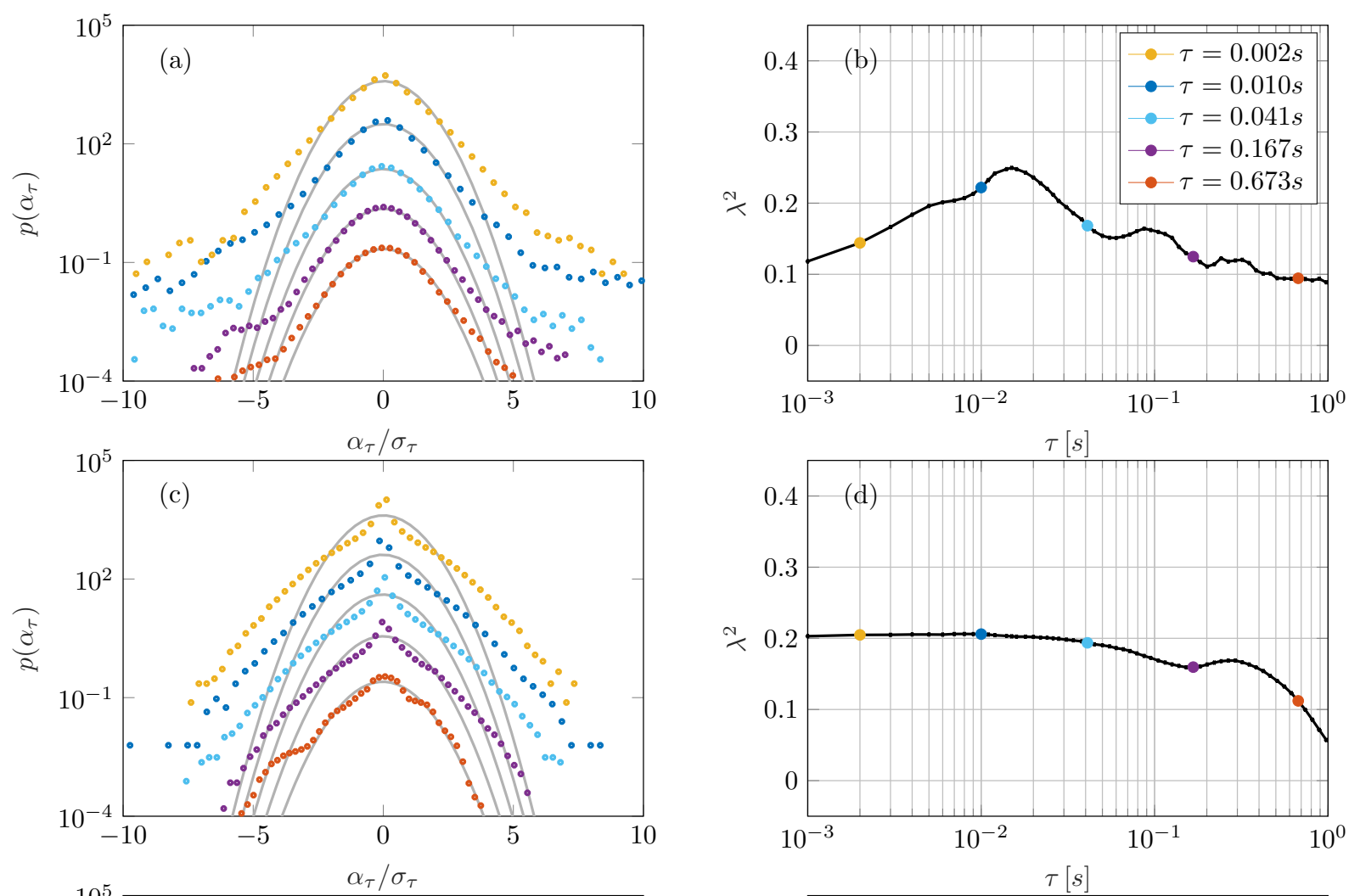

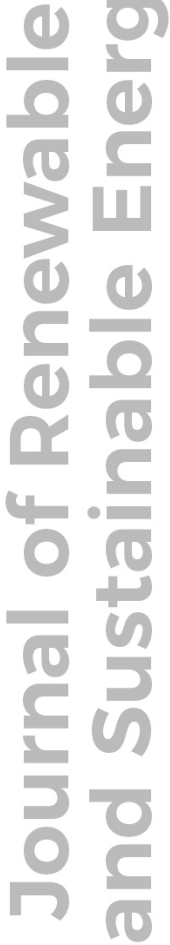
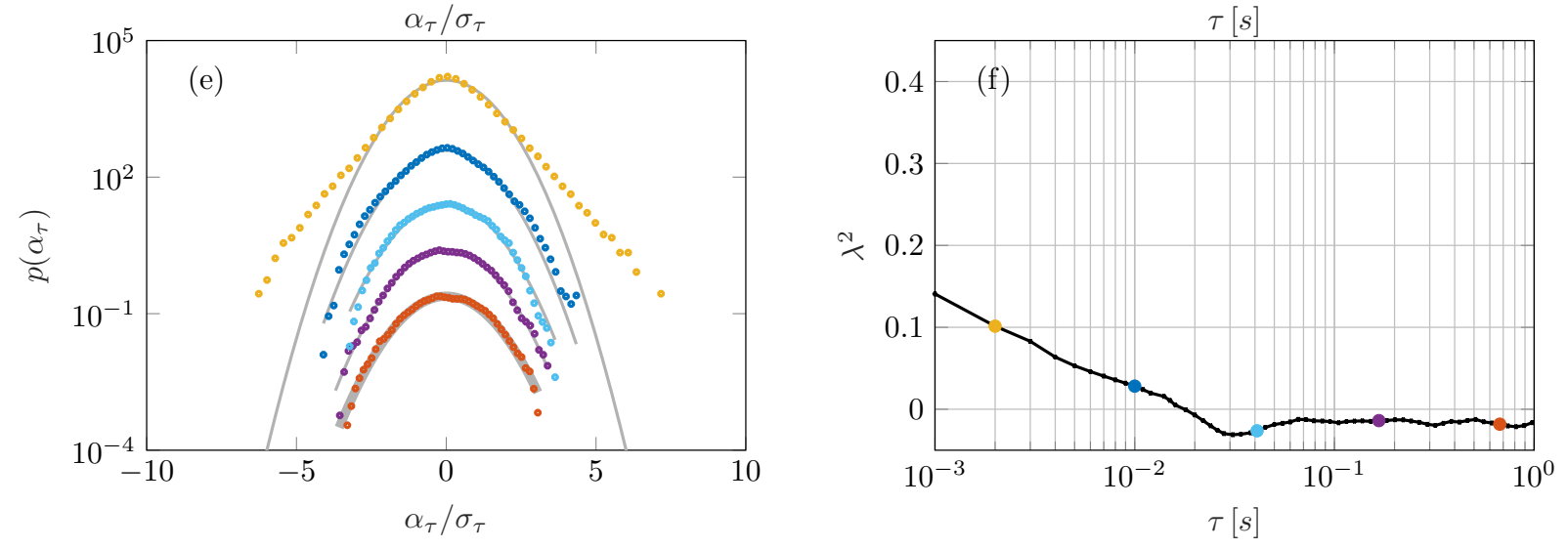


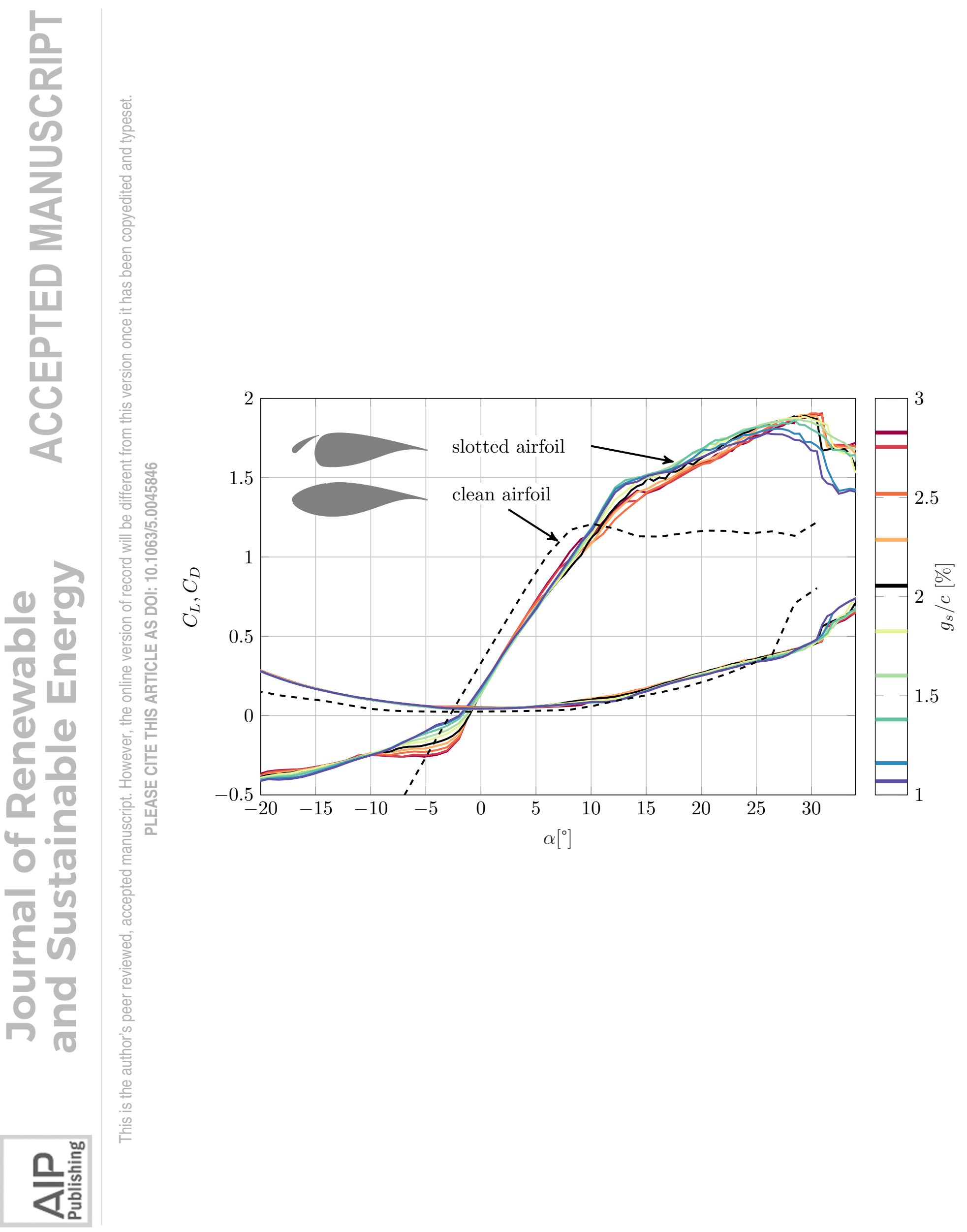




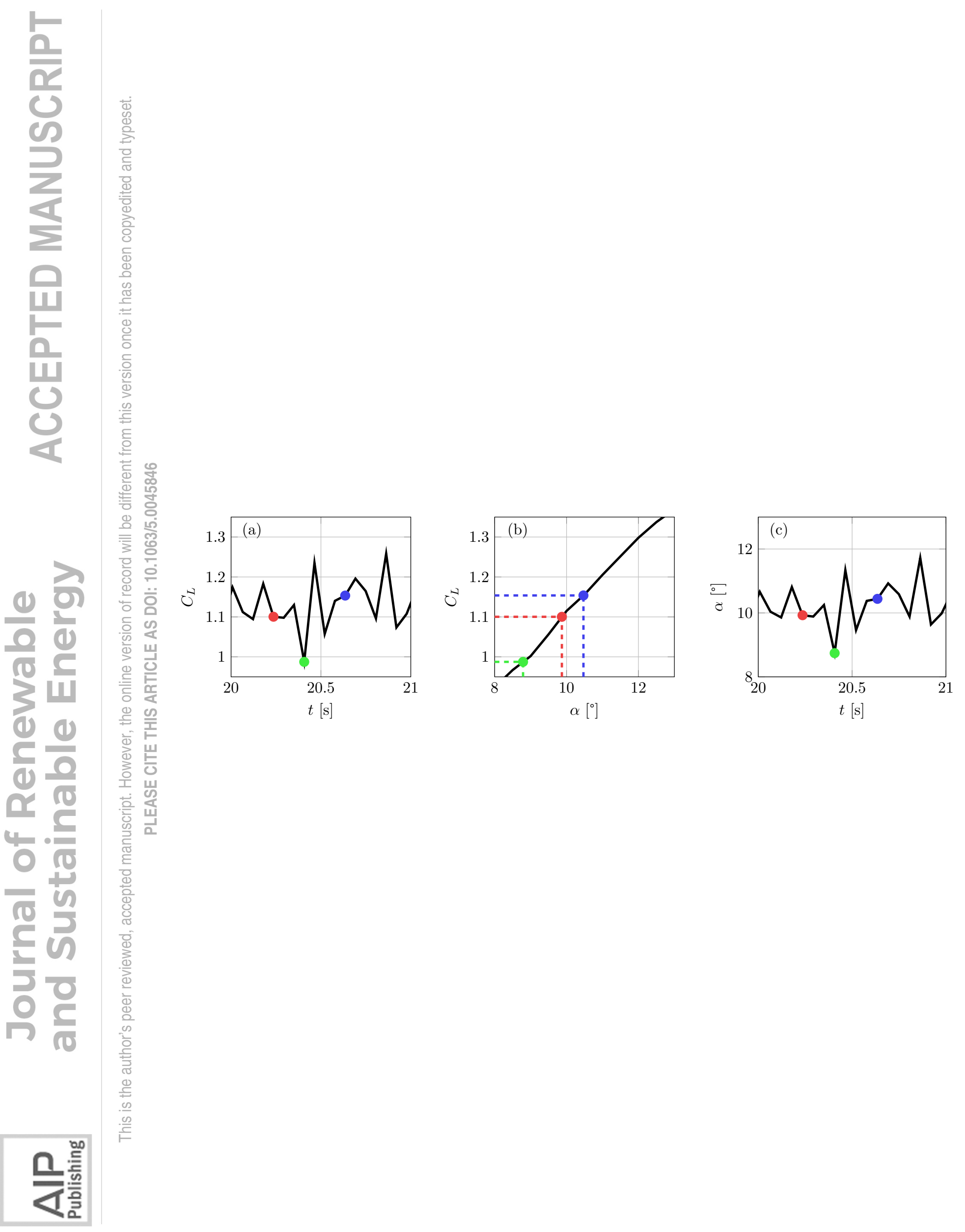




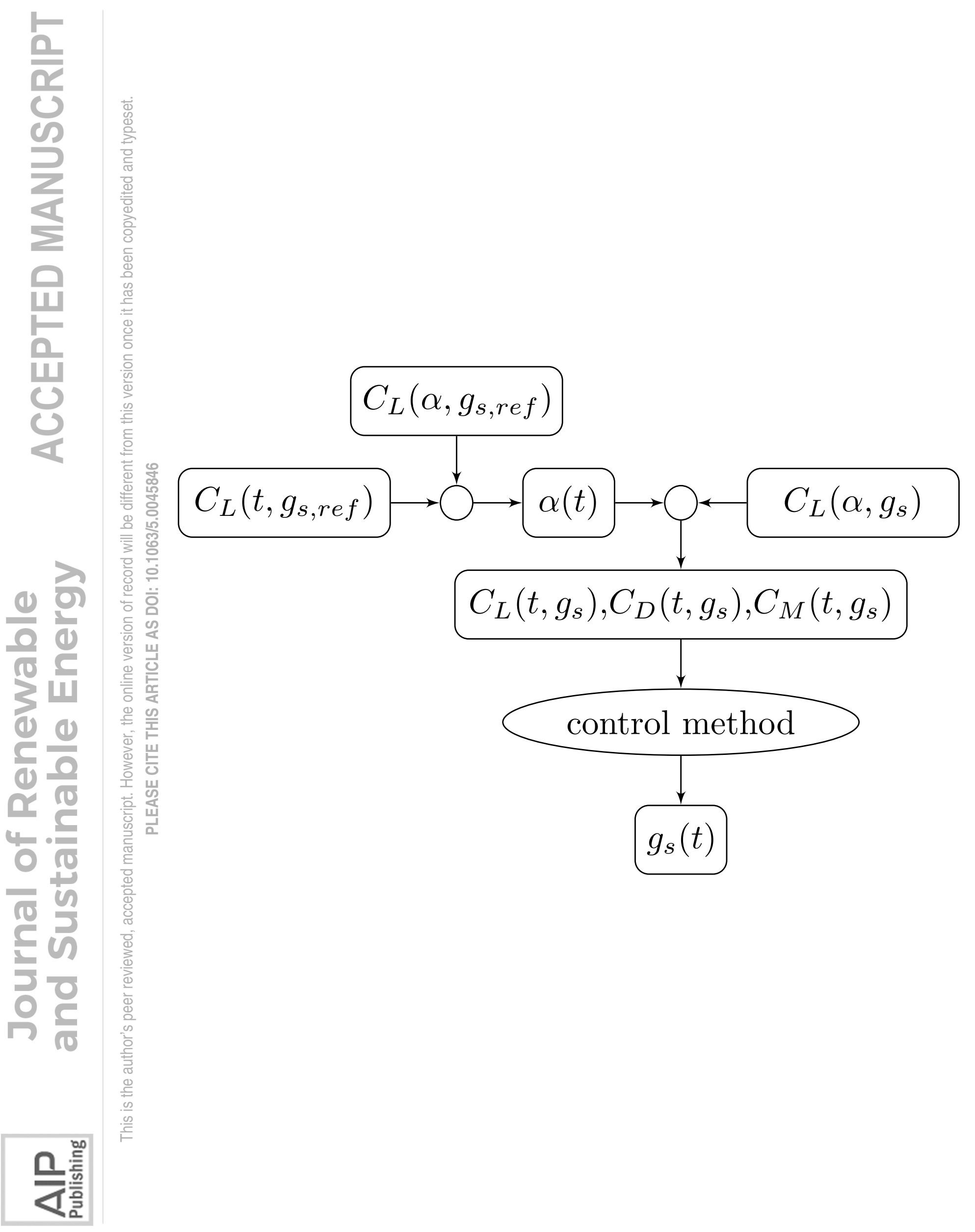




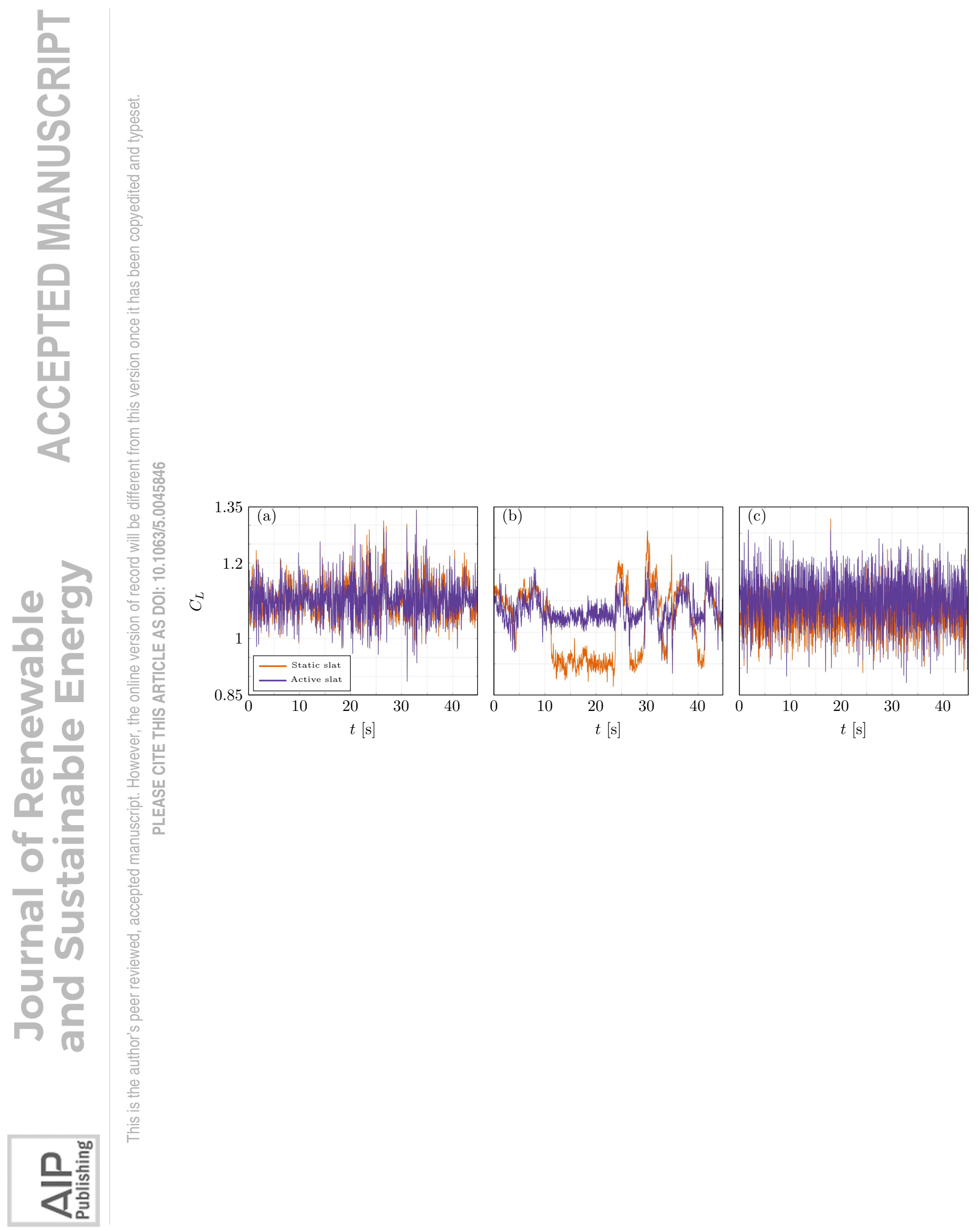




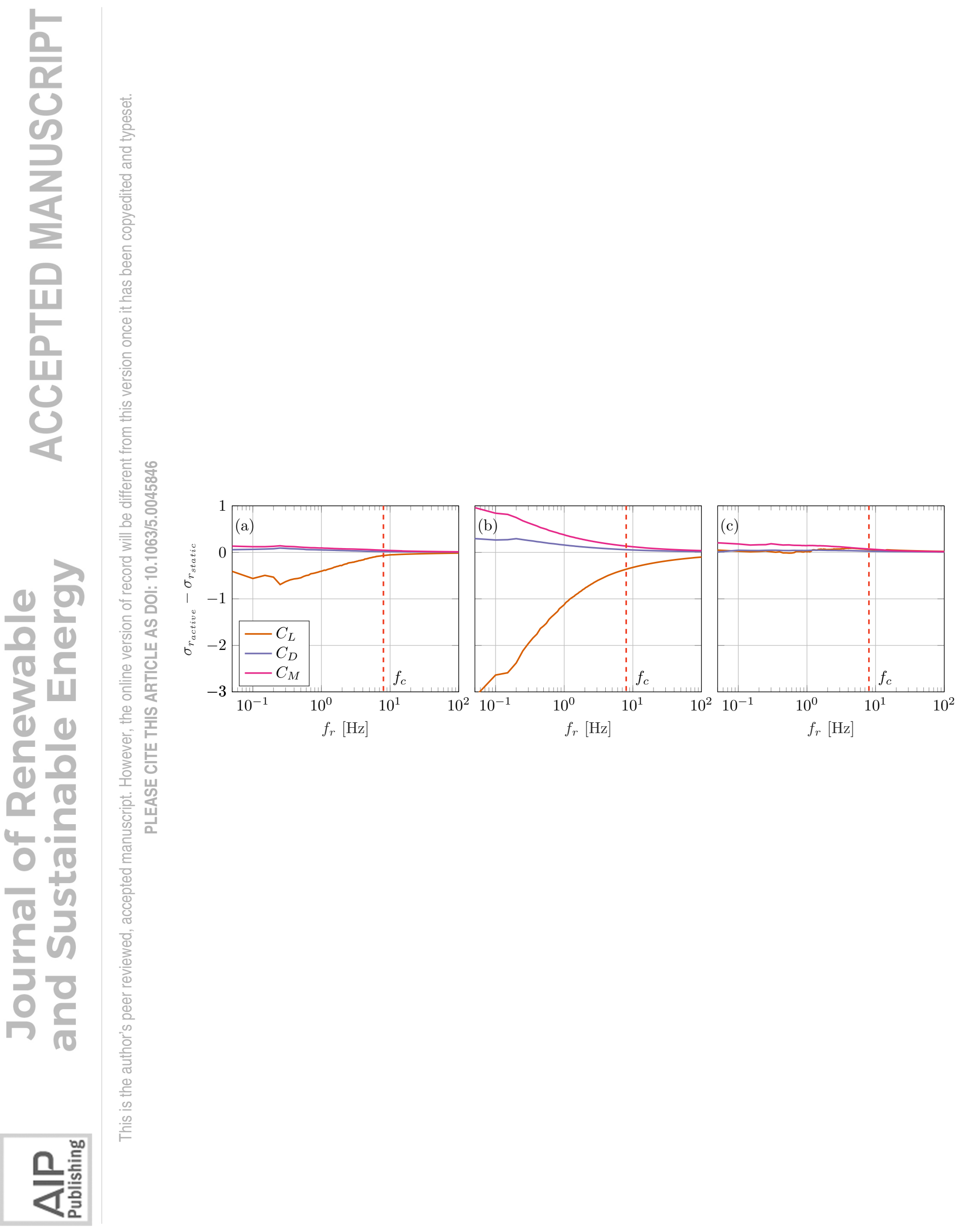




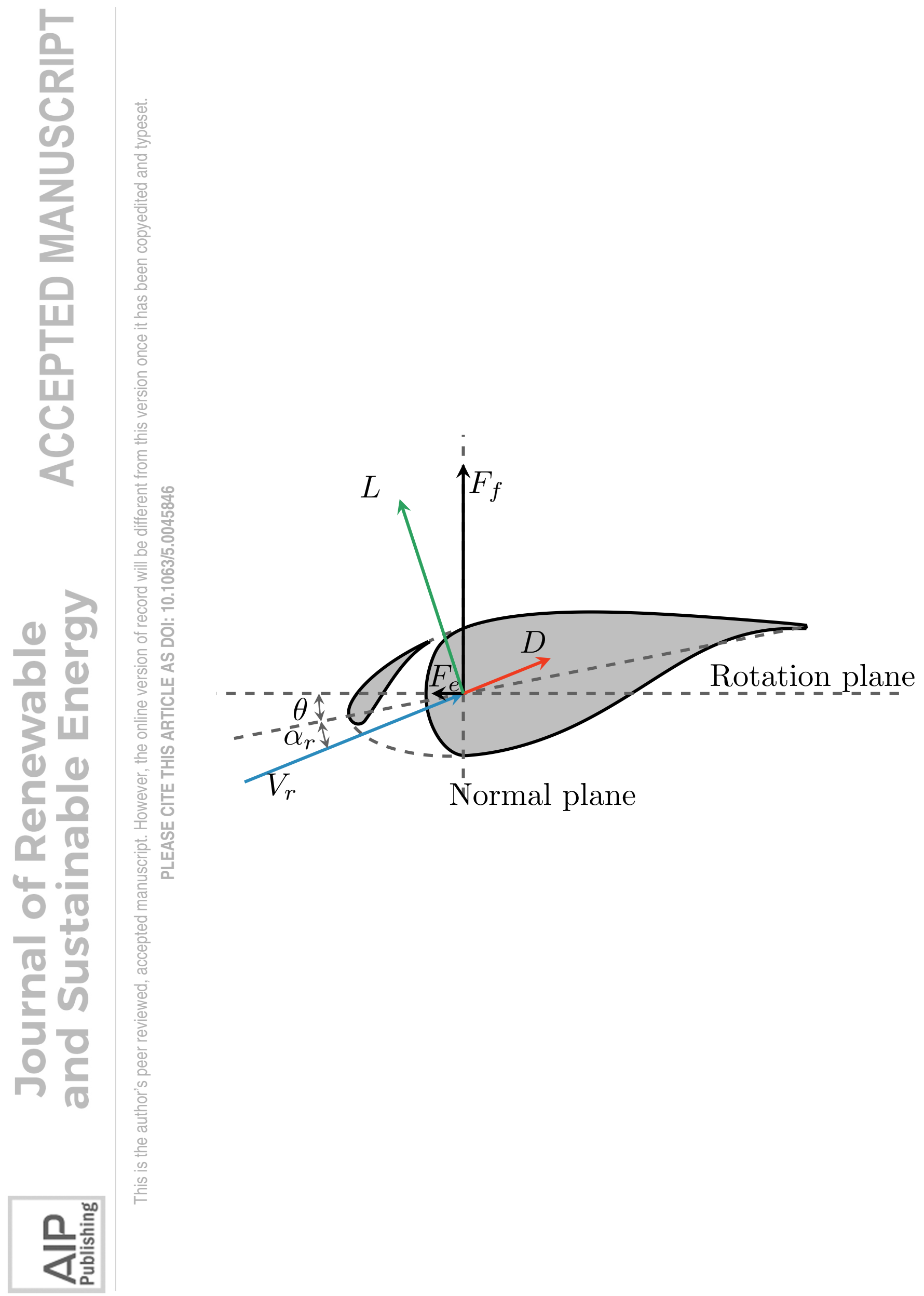



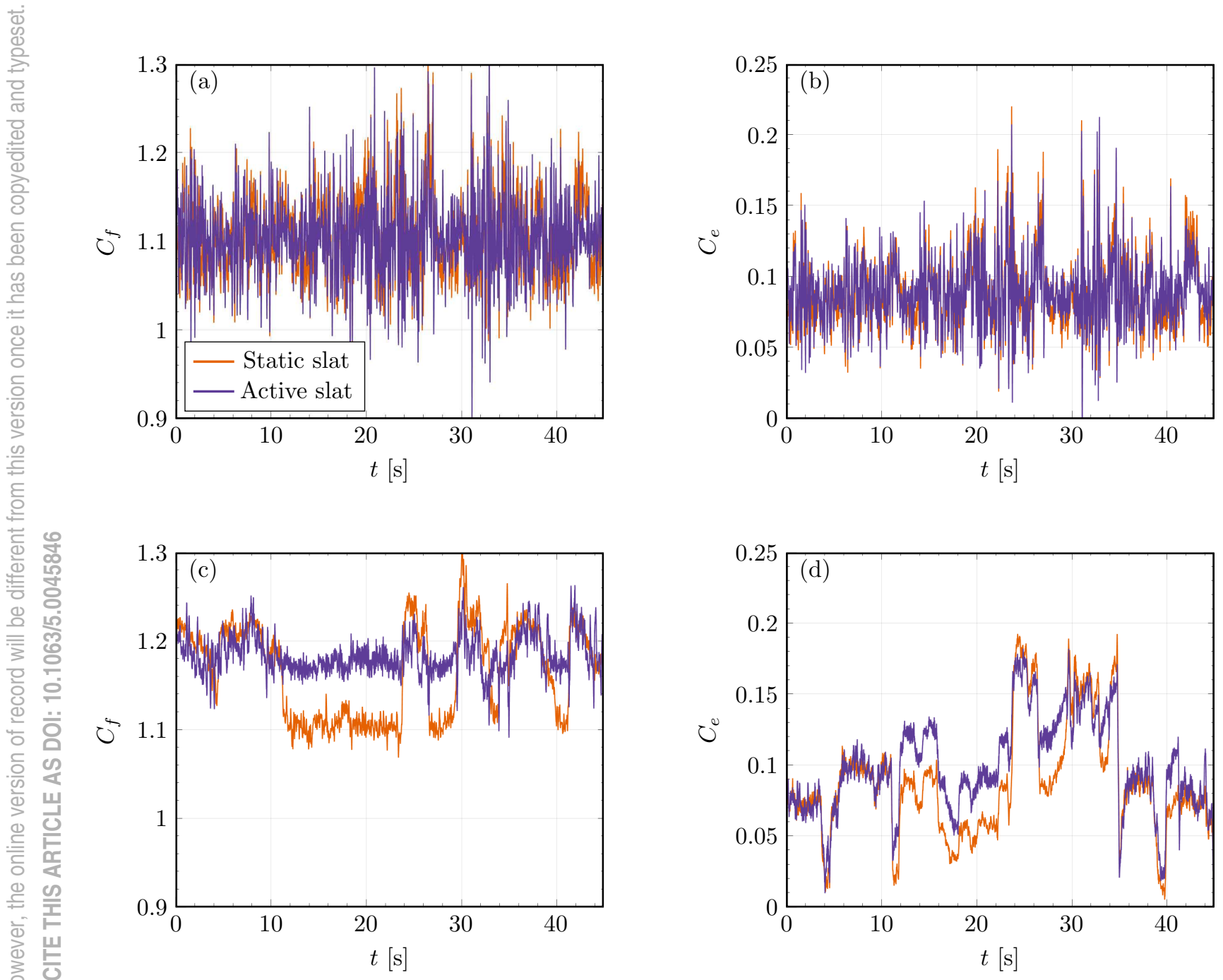

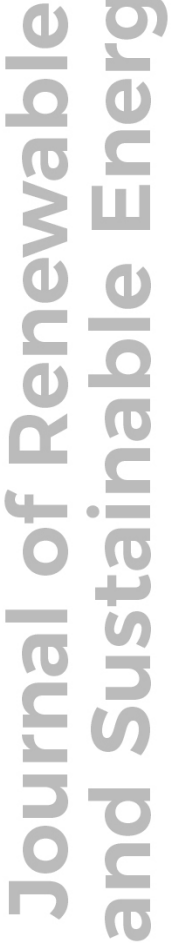

造

픈 눈

을 님
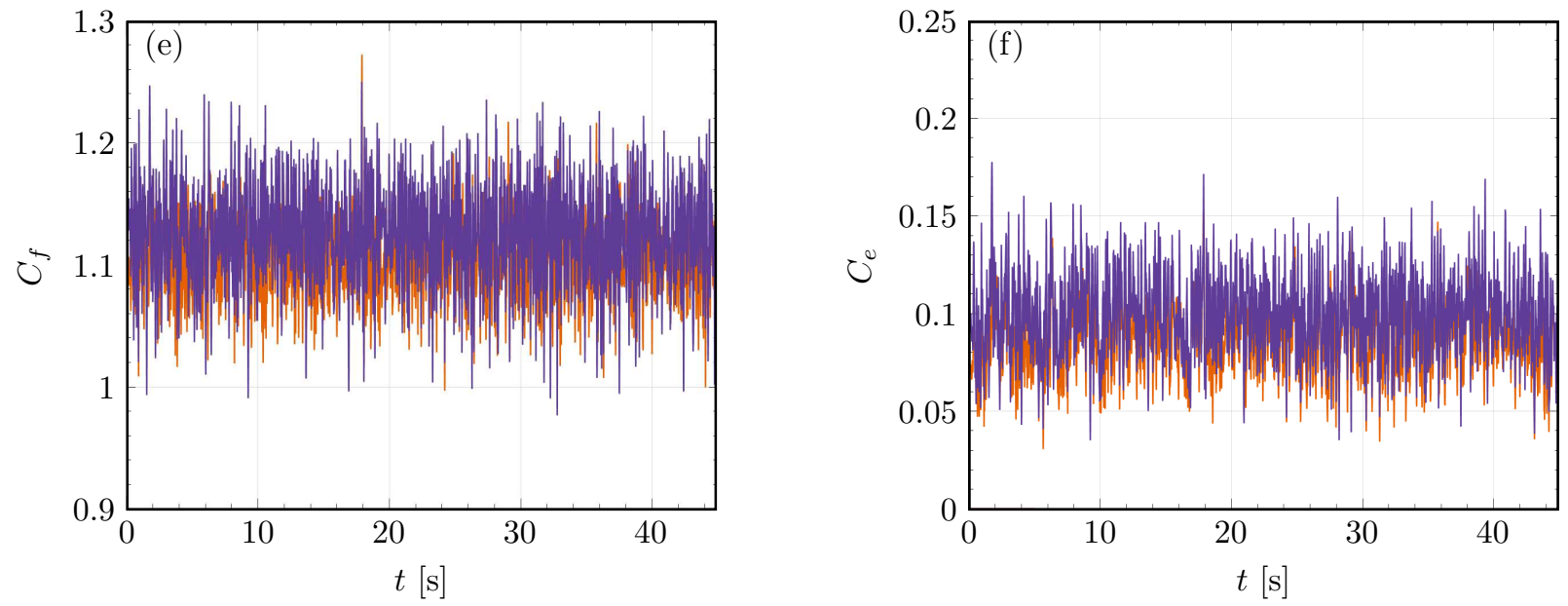

는 